\title{
1 Sclerotinia sclerotiorum hijacks copper from its host for infection
}

2 Yijuan Ding ${ }^{1,2^{*}}$, Jiaqin Mei ${ }^{1,2^{*}}$, Yaru Chai ${ }^{1,2}$, Wenjing Yang ${ }^{1,2}$, Yi Mao ${ }^{1,2}$, Baoqin Yan ${ }^{1,2}$, Yang Yu ${ }^{3}$,

3 Joseph Onwusemu Disi ${ }^{4}$, Kusum Rana ${ }^{1,2}$, Jiana $_{\mathrm{Li}^{1,2}}{ }^{1,2}$ Wei Qian ${ }^{1,2 * *}$

4

$5{ }^{1}$ College of Agronomy and Biotechnology, Southwest University, Chongqing 400715, China

$6 \quad{ }^{2}$ Academy of Agricultural Sciences, Southwest University, Chongqing 400715, China

$7 \quad{ }^{3}$ College of Plant Protection, Southwest University, Chongqing 400715, China.

$8{ }^{4}$ Department of Entomology, University of Georgia, Athens, GA 30602, United States.

$9 \quad$ *These authors contributed equally to this work.

$10 \quad{ }^{* *}$ Correspondence: qianwei666@ hotmail.com. 


\section{Abstract}

Sclerotinia sclerotiorum induces host reactive oxygen species (ROS) production, which leads to necrosis in the host, allowing the pathogen to absorb nutrients from the dead tissues. Here, we found that three $S$. sclerotiorum genes involved in copper ion import/transport, SsCTR1, SsCCS and SsATX1, were significantly up-regulated during infection of Brassica oleracea. Function analysis revealed that these genes involved in fungal ROS detoxification, oxalic acid production, pathogen establishment and virulence. On the host side, four genes putatively involved in copper ion homeostasis, BolCCS, BolCCH, BolMT2A and BolDRT112, were significantly down-regulated in susceptible B. oleracea, but stably expressed in resistant $B$. oleracea during infection. Their homologs were found to promote resistance to necrotrophic pathogens and increase antioxidant activity in Arabidopsis thaliana. Furthermore, copper concentration analysis indicated that copper is transported into the necrotic area from healthy area during infection. Collectively, our data suggest that S. sclerotiorum hijacks host copper to detoxify ROS, whereas the resistant hosts restrict the supply of essential copper nutrients to S. sclerotiorum by maintaining copper ion homeostasis during infection. 


\section{Introduction}

Copper serves as a cofactor in many enzymes and is an essential micronutrient for growth and development of organisms. It is involved in a range of biological processes, including photosynthetic and respiratory electron transport, cell wall remodeling, oxidative stress responses, and ethylene perception (Pilon et al, 2006; Yruela, 2009). Given its importance, copper metabolism has been well-studied in model organisms. In yeast, $\mathrm{Cu}^{2+}$ is reduced to $\mathrm{Cu}^{+}$by cell membrane metalloreductases (Fre1 and Fre2), and $\mathrm{Cu}^{+}$is then transported into cells by the high-affinity $\mathrm{Cu}^{+}$transporters $\mathrm{Ctr} 1$ and Ctr3 (Pena et al, 2000). The Ctr2 transporter mobilizes the stored copper from the vacuole into the cytosol under low-copper conditions (Rees et al, 2004). The cytosolic copper is delivered to the cuproenzymes in diverse ways. For example, the copper homeostasis factor Atx1 binds and delivers copper into Fet3 via the Ccc2 pump in yeast (Lin et al, 1997; Cankorur-Cetinkaya et al, 2016). Copper chaperone CCS delivers copper into $\mathrm{Cu} / \mathrm{Zn}$ SOD in human and yeast (Banci et al, 2012; Gleason et al, 2014), and copper is transferred into cytochrome $c$ oxidase in the mitochondria via copper chaperones such as COX17 and COX11 in eukaryotes (Carr and Winge, 2003; Arnesano et al, 2005). A few genes related to the absorption and distribution of copper have been discovered in Arabidopsis thaliana, such as genes encoding copper transporters (COPTs), chaperone components ( $\mathrm{CCH}, \mathrm{CCS}$ and $\mathrm{COX})$, metallothioneins (MTs), P-type ATPases (HMA, PAA and RAN) and plastocyanin (PETE) (del Pozo et al, 2010; Gu et al, 2015; Abdel-Ghany, 2009).

Reactive oxygen species (ROS) including hydrogen peroxide $\left(\mathrm{H}_{2} \mathrm{O}_{2}\right)$, hydroxyl radical (HO-), singlet oxygen $\left({ }^{1} \mathrm{O}_{2}\right)$ and superoxide anion $\left(\cdot \mathrm{O}_{2}{ }^{-}\right)$, are derived from partial reduction of oxygen $\left(\mathrm{O}_{2}\right)(\mathrm{Liu}$ and $\mathrm{He}$, 2017). ROS have been called 'double-edged swords of life' (Mittler, 2017). On the one hand, ROS act as signaling molecules that regulate development, differentiation, redox levels, stress signaling, interactions with other organisms and systemic responses (Mittler et al, 2011). On the other hand, excess ROS cause 
52

53

54

55

56

57

58

59

60

61

62

63

64

65

66

67

68

69

70

oxidative cellular injury to DNA, RNA, proteins and lipids, and also trigger programmed cell death (Mittler, 2017; Foyer and Noctor, 2013; Mignolet-Spruyt et al, 2016). To avoid or overcome the damage caused by excess of ROS, organisms have developed a complex ROS scavenging system that delicately regulates the balance between production and elimination of ROS. A few cuproenzymes are involved in ROS scavenging and antioxidant activity. For example, the cytosolic $\mathrm{Cu} / \mathrm{Zn}$ superoxide dismutase (Cu/Zn SOD) constitutes the front-line defense against intra- and extracellular ROS (Culotta et al, 2006).

Copper homeostasis factor ATX1 is involved in defense against oxidative stress (Himelblau et al, 1998), while cytochrome $c$ oxidase catalyzes the reduction of oxygen to water in mitochondria (Poyton et al, 1995).

Necrotrophic plant pathogens promote ROS production in the plant host and induce necrosis during host colonization (Heller and Tudzynski, 2011). This raises an interesting question of how necrotrophic plant pathogens survive in such high levels of host-derived ROS. Sclerotinia sclerotiorum is a typical necrotrophic pathogen that causes Sclerotinia stem rot in more than 400 species (Garg et al, 2010). In this study, our data showed that $S$. sclerotiorum hijacks copper from the host and activates ROS detoxification enzymes during infection by enhancing the expression of genes involved in copper ion import and transport, and that resistant hosts limit the supply of copper to $S$. sclerotiorum by maintaining copper ion homeostasis. This research provides new insights into the interaction between S. sclerotiorum and the host, highlighting the importance of ROS and copper in these interactions. 


\section{Results}

\section{Copper is involved in the interaction between Brassica oleracea and S. sclerotiorum}

S. sclerotiorum induces typical lesions, which are the main battlegrounds of gene interactions between $S$. sclerotiorum and the host. We previously detected differentially expressed genes (DEGs) by comparing gene expression in lesions of resistant and susceptible $F_{2}$ plants of B. oleracea (Ding et al, 2019). Here, the set of transcriptome data was analyzed for dynamic changes of gene expression in sclerotinia and hosts during infection. A total of 738 and 228 S. sclerotiorum DEGs (24 hours post inoculation [hpi] vs 12 hpi) were detected in lesions of resistant and susceptible B. oleracea, respectively (Fig EV1A), which were significantly enriched for three overlapping Gene Ontology (GO) terms, 'oxidation-reduction process', 'copper ion transport' and 'copper ion import' (Fig 1A). Eight S. sclerotiorum DEGs involved in the 'copper ion transport' and 'copper ion import' processes were up-regulated during infection as revealed by both RNA-seq analysis and qRT-PCR analysis (Figs EV1B and Appendix Figure S1A).

A total of 5988 and 5441 DEGs (24 hpi vs 12 hpi) were detected and subjected to GO analysis in resistant and susceptible $B$. oleracea stems, respectively (Fig EV1C). Interestingly, the biological process 'copper ion homeostasis' was significantly enriched in susceptible B. oleracea but not in resistant B. oleracea (Fig 1B). Among ten DEGs involved in 'copper ion homeostasis' (Fig EV1D), seven genes (Bol023613, Bol026950, Bol044257, Bol002542, Bol011307, Bol000591 and Bol029708) with consistent expression patterns between RNA-seq analysis and qRT-PCR analysis were significantly down-regulated in susceptible $B$. oleracea plants, but only slightly down-regulated or stably expressed in resistant $B$. oleracea plants (Appendix Figure S1B). We further analyzed their expression in parental resistant (C01) and susceptible (C41) B. oleracea lines via qRT-PCR. All seven genes showed sharply down-regulated expression in susceptible parental line C41, while six of the seven genes (Bol023613, Bol044257, Bol002542, Bol011307, Bol000591 and Bol029708) showed a stably or even slightly 
up-regulated expression in the resistant parental line C01 (24 hpi vs 12 hpi) (Appendix Figure S1C). This suggests that copper ion homeostasis may be disrupted in susceptible $B$. oleracea but not in resistant $B$. oleracea during early infection.

\section{Copper is transported into the necrotic area from healthy area during infection}

We analyzed the copper distribution in and around lesions of infected leaves and stems in the moderately resistant rapeseed (Brassica napus) cultivar Zhongshuang 11 at $48 \mathrm{hpi}$. The content of copper in the necrotic areas $\left(\mathrm{Cu}_{\text {leaf-N }}=11.76 \mu \mathrm{g} / \mathrm{g}, \mathrm{Cu}_{\text {stem-N }}=4.00 \mu \mathrm{g} / \mathrm{g}\right)$ was significantly higher than that in the uninfected $\left(\mathrm{Cu}_{\text {leaf }-\mathrm{U}}=8.90 \mu \mathrm{g} / \mathrm{g}, \mathrm{Cu}_{\text {stem-U }}=2.94 \mu \mathrm{g} / \mathrm{g}\right)$ and margin $\left(\mathrm{Cu}_{\text {leaf-Ma }}=10.05 \mu \mathrm{g} / \mathrm{g}, \mathrm{Cu}_{\text {stem-Ma }}=3.33\right.$ $\mu \mathrm{g} / \mathrm{g})$ areas in both leaves and stems $(P<0.05)$, and significant differences in copper content were found in necrotic areas compared to mock-infected tissue $\left(\mathrm{Cu}_{\text {leaf-Mock }}=9.09 \mu \mathrm{g} / \mathrm{g}, \mathrm{Cu}_{\text {stem-Mock }}=3.29 \mu \mathrm{g} / \mathrm{g}\right)(P<$ 0.05) (Fig 1C). These data indicate that copper is transported into the necrotic area from healthy area during early infection.

\section{Copper ion homeostasis genes promote host resistance}

To test whether copper ion homeostasis is associated with host resistance, the six genes in this process that were stably expressed or slightly up-regulated in resistant $B$. oleracea but significantly down-regulated in susceptible $B$. oleracea were aligned with four A. thaliana orthologs (AtCCS, AtMT2A, AtDRT112 and AtCCH) (Appendix Figure S2A). We tested the function of these A. thaliana homologs with respect to $S$. sclerotiorum resistance using T-DNA mutants (atccs, atmt2a, atdrt112 and atcch) and overexpression lines (OX-AtCCS, OX-AtMT2A, OX-AtDRT112 and OX-AtCCH) (Appendix Figure S2B and C). Notably, all of the reduced-expression mutant lines were more susceptible to S. sclerotiorum compared to the wild type, while the overexpression lines displayed higher resistance (Fig 2A). At $24 \mathrm{hpi}$, the lesion size was $1.28-1.35 \mathrm{~cm}^{2}$ in the T-DNA mutant lines, $1.06-1.07 \mathrm{~cm}^{2}$ in the wild type and $0.59-0.62 \mathrm{~cm}^{2}$ in the overexpression lines (Fig $2 \mathrm{~B}$ ). 


\section{Copper ion homeostasis is associated with response to oxidative stress in the host}

118 To further explore how copper ion homeostasis is associated with host resistance, the transcriptomes of

119 leaves from $A$. thaliana wild type, T-DNA mutants and overexpression lines of AtCCS, AtMT2A, AtDRT112 and $A t C C H$ at 0, 6, and 12 hpi were sequenced, producing an average of 22.9 million clean reads for each sample. On average, 17.7 and 0.97 million clean reads were mapped to the reference genome of $A$. thaliana and $S$. sclerotiorum per sample, respectively. As expected, the overexpression lines exhibited higher expression of the corresponding target genes than the T-DNA mutants and the wild type line (Fig EV2A). Five common GO terms, including 'response to oxidative stress', were detected by comparing the up-regulated DEGs between overexpression lines and T-DNA mutants and between overexpression lines and wild type at $12 \mathrm{hpi}$ (Fig 3A). Considering that more DEGs were found between the overexpression lines and T-DNA mutants than between the overexpression lines and wild type, we conducted a Weighted Gene Co-expression Network Analysis (WGCNA) using a total of 7321

A. thaliana DEGs between the overexpression lines and T-DNA mutants (Table EV1). This analysis produced 17 modules (groups of genes with similar expression pattern), of which one (shown in red) showed a negative correlation with lesion size $(r=-0.45, P=0.03)$ (Fig EV2B). It encompassed 394 genes, which were significantly enriched for the biological process of 'response to oxidative stress' (Fig 3B). In addition, total of 1273 S. sclerotiorum DEGs (overexpression lines vs. T-DNA mutants) were detected and used for WGCNA, which resulted in five significant modules $(P<0.05)$, two of which were highly correlated with peroxisome organization and oxidation-reduction (Table EV2; Fig EV2C). we analyzed the antioxidant activity of these overexpression lines and T-DNA mutants by staining inoculated leaves at 0, 6 and 12 hpi with 3,0-diaminobenzidine (DAB) and nitrotetrazolium blue 
chloride (NBT) for the accumulation of $\mathrm{H}_{2} \mathrm{O}_{2}$ and $\cdot \mathrm{O}_{2}^{-}$, respectively. We observed deeply stained areas around the inoculant column at both 6 and $12 \mathrm{hpi}$ in the T-DNA mutant lines, but more lightly stained areas in the overexpression lines (Fig 4A), suggesting that antioxidant activity was enhanced in the overexpression lines but suppressed in the T-DNA mutants. This conclusion was further supported by a $\mathrm{Cu} / \mathrm{Zn}$ SOD enzyme activity assay, in which the overexpression lines exhibited 41.79- to 45.93 and 49.62 to $66.97 \mathrm{U} / \mathrm{mgprot}$, and T-DNA mutants exhibited 14.43 to 22.03 and 10.71 to $26.77 \mathrm{U} / \mathrm{mgprot}$ enzyme activity at 6 and 12 hpi, respectively (Fig 4B). Wild type line was in the middle with 32.14 and 42.82 U/mgprot at 6 and $12 \mathrm{hpi}$, respectively. These results indicate that copper ion homeostasis is associated with the detoxification of ROS in the host.

Considering that necrotrophic pathogens exploit the host oxidative burst, which contributes to pathogen attack (Heller and Tudzynski, 2011; Govrin and Levine, 2000), we hypothesized that copper ion homeostasis was associated with host resistance against diverse necrotrophic fungal pathogens. To test this hypothesis, we examined host resistance against another typical necrotrophic pathogen, Botrytis cinerea (Amselem et al, 2011). The Arabidopsis AtCCS, AtMT2A, AtDRT112 and AtCCH overexpression lines increased resistance to $B$. cinerea by $30-60 \%$, whereas the corresponding T-DNA mutants reduced resistance by 20-30\% in comparison with wild type at 24 hpi (Fig EV3).

\section{S. sclerotiorum requires trace copper for pathogen infection}

Copper is an essential nutrient for microbial pathogens and serves as an important cofactor of enzymes that scavenge ROS (Banci et al, 2012; Carr and Winge, 2003). To test the hypothesis that $S$. sclerotiorum takes up and utilizes host-derived copper ions for the synthesis or activation of enzymes involved in ROS scavenging during infection, we explored the roles of three S. sclerotiorum DEGs annotated as involved in 'copper ion transport/import': SS1G_05578 (SsCTR1), SS1G_00102 (SsCCS) and $S S 1 G_{-} 10888$ (SSATX1) with their silenced and overexpressing strains (Fig EV4A and B). The 
163 products of these genes function to transport extracellular copper into the fungal cell (SsCTRI), deliver 164 copper to $\mathrm{Cu} / \mathrm{Zn} \mathrm{SOD}(S s C C S)$ and detoxify the oxidative damage (SsATX1) (Cobine et al, 2006; Ding 165 et al, 2011; Himelblau et al, 1998). These S. sclerotiorum genes exhibited down-regulated expression in 166 all of the silenced strains (Sictr1, Siccs and Siatx1), and up-regulated expression in all of the 167 overexpressing strains (OXctr1, OXccs and OXatx1) (Fig 5A), indicating they coordinately expressed in 168 S. sclerotiorum. The lesion size after inoculation with the silenced strains was smaller than those after 169 inoculation with the wild-type strain, while the largest lesion size was observed after inoculation with 170 the overexpressing strains in the detached leaves of B. napus and Arabidopsis seedlings (Fig EV4C-E), 171 indicating that these genes are involved in the virulence of S. sclerotiorum.

172 Copper content in the leaves infected by the silenced and overexpressing strains of these three genes 173 was determined. All the strains showed higher copper content in the necrotic areas than the uninfected 174 and margin tissues (Fig 5B). The content of copper in the necrotic areas of overexpressing strains 175 contained $7.90 \%-10.93 \%$ more copper than that of wild-type, and the content of copper in the necrotic 176 areas of silenced strains contained $11.62 \%-16.38 \%$ less copper than that of wild-type (Fig 5B). These 177 results suggest that $S s C T R 1, S s C C S$ and $S s A T X 1$ may associated with copper uptake in S. sclerotiorum 178 during infection. To test the role of copper in the virulence of $S$. sclerotiorum, we sprayed 179 low-concentration $\mathrm{CuSO}_{4}$ solutions $(0.01,0.025$ and $0.05 \mathrm{mg} / \mathrm{L})$ onto the leaf surface and incubated for 18030 min prior to inoculation with each of the silenced strains. The lesion sizes after inoculation with the 181 three silenced strains increased by 1.1 - to 1.4 -fold at 48 hpi in comparison with the non-CuSO 4 182 treatment (sprayed with $\mathrm{ddH}_{2} \mathrm{O}$ ), but exhibited no significant difference from the treatment that was 183 sprayed with $\mathrm{ddH}_{2} \mathrm{O}$ and inoculated with the wild-type strain 1980 (Fig 6A and B). However, treatment 184 with highly concentrated $\mathrm{CuSO}_{4}$ solutions $(0.1$ and $0.25 \mathrm{mg} / \mathrm{L})$ significantly reduced disease symptoms 185 of the silenced strains $(P<0.05)$ (Fig 6B). Similar observations were detected in the wild-type and 
overexpressing stains, which increased lesion size under low concentration of $\mathrm{CuSO}_{4}$, but decreased lesion size in high concentration of $\mathrm{CuSO}_{4}$ (Fig 6B). We further found that low-concentration $\mathrm{CuSO}_{4}$ promoted the growth of all the stains on potato dextrose agar (PDA) plates, and that high-concentration $\mathrm{CuSO}_{4}$ suppressed the growth of S. sclerotiorum (Fig 6C). Especially, the growth inhibition was higher in the silenced strains than the overexpressing strains in high-concentration $\mathrm{CuSO}_{4}(0.25 \mathrm{mg} / \mathrm{L})$ (Fig 6C). These findings suggest that low-concentration copper could promote the growth of strains, and restores the virulence of the silenced strains. It indicates that trace copper is required for the growth and infection of S. sclerotiotum.

\section{Copper ion transport/import involves in ROS scavenging in $S$. sclerotiorum}

To further test the hypothesis that S. sclerotiorum utilizes copper to promote ROS scavenging, we observed superoxide $\left(\cdot \mathrm{O}_{2}^{-}\right)$accumulation among wild-type strain 1980, the silenced strains and overexpressing strains of $S s C T R 1, S s C C S$ and $S s A T X 1$ using NBT staining. The accumulation of $\cdot \mathrm{O}_{2}^{-}$in the fungal hyphal tips was highest in the silenced strains, followed by the wild-type strain and the overexpressing strains (Fig 7A). We then cultured all strains on PDA supplemented with different concentrations of $\mathrm{H}_{2} \mathrm{O}_{2}(0,2,6,10$ and $15 \mathrm{mM})$ (Fig 7A). The growth of the silenced strains was most seriously inhibited (reduced by $26.2-100 \%$ ), followed by the wild-type strain (reduced by 18.0-90.3\%) and the overexpressing strains (reduced by 2.2-68.4\%) (Fig 7B). These observations indicate that ROS can inhibit the growth of S. sclerotiorum and that these S. sclerotiorum genes are involved in the detoxification of ROS.

Because $\mathrm{Cu} / \mathrm{Zn}$ SOD is one of the primary superoxide scavengers (Mittler, 2017), we analyzed the SSSOD1 expression level in the wild-type, silenced, and overexpressing strains. In the silenced strains, SSSOD1 transcript accumulation was only 0.5-0.7 times the wild-type levels, while the overexpressing strains showed higher of SSSOD1 expression, by 1.7- to 2.1-fold relative to wild-type strain (Fig 7C). In 
comparison with the wild-type (572.51 U/gFW), the $\mathrm{Cu} / \mathrm{Zn}$ SOD enzyme activity was lower in silenced strains (372.88-499.39 U/gFW) and higher in overexpressing strains (750.44-909.86 U/gFW) (Fig 7D).

These results indicate that these three $S$. sclerotiorum copper-related genes promote the expression of SsSOD1 and increase enzyme activity of Cu/Zn SOD, resulting in the increase of ROS scavenging capacity in the fungal cells.

\section{Copper ion transport/import genes promote infection cushions formation and oxalic acid} production in S. sclerotiorum

ROS is associated with the formation of infection cushions and production of oxalic acid in $S$. sclerotiorum (Liberti et al, 2013; Veluchamy et al, 2012; Kim et al, 2011). Infection cushions are essential for the S. sclerotiorum to penetrate the host cuticle and infect plants (Liang and Rollins, 2018), and oxalic acid (OA) is one of the virulence factors of S. sclerotiorum (Li et al, 2018; Liang and Rollins, 2018). Therefore, we monitored the infection cushions and OA production among the wild-type, silenced and overexpressing strains on B. napus leaves. Compared to the wild-type strain, the silenced strains exhibited fewer and looser infection cushions, while overexpressing strains exhibited more and compacter infection cushions (Fig EV5A), indicating that these S. sclerotiorum genes affect the establishment of infection.

Meanwhile, we detected significant differences in OA concentration among the wild-type, silenced and overexpressing strains after culturing for 2 days on PDA medium. The average OA concentration in the silenced strains was $6.30 \mathrm{mg} / \mathrm{ml}$, followed by the wild-type $(7.81 \mathrm{mg} / \mathrm{ml})$ and overexpressing strains $(9.39 \mathrm{mg} / \mathrm{ml})($ Fig EV5B). At the same time, the average $\mathrm{pH}$ value of the culture medium from the overexpressing strains $(\mathrm{pH}=2.79)$ was lowest, followed by the wild-type $(\mathrm{pH}=2.92)$ and silenced strains $(\mathrm{pH}=3.13)($ Fig EV7C). The OA synthesis gene $S s O A H$ exhibited lower expression in the silenced strains than in the wild-type strain, but higher expression in the overexpressing strains (Fig 
232 EV5D). It suggests that expression disruption of the copper ion transport/import genes suppresses the

233 OA production in S. sclerotiorum.

\section{Discussion}

235 Copper is a component of numerous enzymes and plays a key role in the responses to oxidative stress

236 (Culotta et al, 2006; Berterame et al, 2018). By analyzing differentially expressed genes (DEGs) at

237 lesion for dynamic changes in host and S. sclerotiorum during infection, we found that the genes in the

238 'copper ion import' and 'copper ion transport' with up-regulated expression involved in S. sclerotiorum

239 copper uptake, virulence, ROS detoxification, fungal establishment and OA production, and that the host

240 genes in the 'copper ion homeostasis' with stable expression in the resistant line, but down-regulated

241 expression in the susceptible line were associated with response to oxidative stress and resistance to $S$.

242 sclerotiorum. Our data indicate a battlefield at the host-S. sclerotiorum interface where S. sclerotiorum

243 hijacks host-derived copper to detoxify ROS in its cell, while the resistant host maintains 'copper ion

244 homeostasis' to limit the supply of copper to the pathogen.

245 The generation of ROS at the infection site is one of the earliest responses of pathogen-associated 246 molecular-pattern-triggered immunity (PTI) (Jones and Dangl, 2006). As secondary messengers, ROS 247 are indispensable for signaling, stress responses and developmental processes (Marschall and Tudzynsk, 248 2014). However, excess ROS trigger programmed cell death (PCD) and cause host necrosis, which 249 facilitates the growth of necrotrophic pathogens (Heller and Tudzynski, 2011; Kim et al, 2008; Lu and 250 Higgins, 1999). Antioxidant components in the host, such as peroxidase, SODs, glutathione sulfhydryl 251 transferase (GST) and glutathione (GSH) are associated with resistance against S. sclerotiorum (Liang et 252 al, 2008; Yang et al, 2007; Wen et al, 2013; Wei et al, 2016; Mei et al, 2016; Garg et al, 2013), and 253 resistance against $S$. sclerotiorum can be improved by decreasing the accumulation and/or production of 254 ROS in the host (Ranjan et al, 2018; Wang et al, 2014). The four DEGs in the biological process of 
255 'copper ion homeostasis' we detected during infection are involved in various antioxidant activities.

$256 A t C C S$ is responsible for the activation of $\mathrm{Cu} / \mathrm{Zn}$ SOD (Chu et al, 2005). Metallothioneins (MTs), which

257 act as heavy metal chelators and ROS scavengers, contribute to plant adaptation to abiotic stresses (Kim 258 and Kang, 2018). DRT112 is one of two Arabidopsis plastocyanin genes (PETE2), which function to 259 buffer excess copper (Abdel-Ghany, 2009). AtCCH is a homolog of yeast ATX1, which functions to 260 deliver copper into laccase or Fet3 (Himelblau et al, 1998). In this study, we found that the 261 overexpression of these genes in Arabidopsis enhanced host ROS detoxification and resistance against $S$. 262 sclerotiorum, and the genes in the biological process of 'copper ion homeostasis' were coordinately 263 expressed with those for 'response to oxidative stress'. Therefore, our research provides evidence that copper ion homeostasis is associated with ROS detoxification in host.

265 Free copper ions induce ROS production (Rae et al, 1999), which may be toxic to S. sclerotiorum. We 266 found that highly concentrated $\mathrm{CuSO}_{4}$ solutions suppressed the growth of S. sclerotiorum. In fact, 267 copper is one of important active ingredients in many bactericidal and fungicidal agents, such as 268 Bordeaux mixture (Martins et al, 2014). However, S. sclerotiorum can grow in necrotic areas with a 269 relatively high concentration of copper. Three aspects of our findings might help explain this 270 phenomenon. (1) The copper uptake system of S. sclerotiorum was elevated during infection. SsCTR1, 271 which functions to import extracellular copper into the fungal cells (Samanovic et al, 2012), exhibited 272 up-regulated expression, indicating that host-derived copper may be imported into the S. sclerotiorum 273 cells. (2) A few genes associated with copper detoxification, such as MTs (SsMT), Sur7 (SsSUR7) and 274 P-type ATPase (SsATP7A) (Weissman et al, 2000; Douglas et al, 2012; Ladomersky and Petris, 2015), 275 were not significantly induced in S. sclerotiorum during infection (Appendix Figure S3), indicating that 276 the amount of host-derived copper was not high enough to produce toxic effects in S. sclerotiorum. (3) 277 SsCCS, SsCOX17 and SsATX1 expression was up-regulated during infection. The homologs of SsCCS 
function to deliver copper to oxidative scavengers Cu/Zn SOD (Gleason et al, 2014; Cobine et al, 2006).

The ATX1 gene could act as a multi-copy suppressor of oxidative damage in yeast (Himelblau et al, 1998). It indicated that the copper might be utilized for the synthesis or activation of these ROS detoxification enzymes of $S$. sclerotiorum during infection. Thus, this study provides important insights into the question about survival of S. sclerotiorum at relatively high levels of ROS.

The idea of 'nutritional immunity' was first proposed to describe the resistance mechanism in human cells wherein they withhold transition metals, such as iron, zinc, manganese and copper to defend against microbial pathogen invaders (Besold et al, 2016; Malavia et al, 2017; Hood and Skaar, 2012; Crawford and Wilson, 2015). To overcome this strategy, successful pathogenic species must evolve specialized mechanisms to adapt to the nutritionally restrictive environment of the host and cause disease. For example, the human kidney and brain can thwart Candida albicans growth by limiting the supply of copper nutrients, producing 'copper starvation' to C. albicans (Besold et al, 2016). In response, C. albicans induces copper uptake machineries that enable it to survive in a copper-starved environment (Li et al, 2015). Here, the defense response in resistant lines that stabilized the expression of copper ion homeostasis genes and limited the availability of copper to S. sclerotiorum may be considered a form of nutritional immunity. To the best of our knowledge, this is the first description of nutritional immunity in plants. We therefore propose a possible host-S. sclerotiorum interaction model in which resistant plants induce nutritional immunity and restrict the supply of essential copper nutrients to $S$. sclerotiorum by maintaining copper ion homeostasis, while S. sclerotiorum enhances its copper uptake system and hijacks host-derived copper, which activates its ROS scavenging system during infection and promotes its survival and virulence (Fig. 8).

\section{Methods and materials}




\section{Experimental strains and plants}

302 The wild-type strain of S. sclerotiorum 1980 and B. cinerea wild-type strain B05.10 (Amselem et al, 303 2011) were used in this study. Fungal strains were grown on potato dextrose agar (PDA, 20\% potato, 2\% 304 dextrose and $1.5 \%$ agar) at $22^{\circ}$ C. S. sclerotiorum transformants were cultured on PDA amended with 80 $305 \mu \mathrm{g} / \mathrm{ml}$ hygromycin B (Calbiochem, San Diego, CA) to stabilize the transformants. Seedlings from $A$. 306 thaliana Col-0 (ecotype Columbia-0), T-DNA mutants and overexpression transgenic lines were grown 307 in the autoclaved soil (Pindstrup) at $20 \pm 2{ }^{\circ} \mathrm{C}$ under a $12 \mathrm{~h}$ light/dark cycle with $70 \%$ relative humidity. 308 The rapeseed cultivar Zhongshuang 11 was used for virulence assay.

Vector construction and transformation of $S$. sclerotiorum and A. thaliana

The fragments of four S. sclerotiorum genes (SsATX1: 221 bp, SsCOX17: 232 bp, SsCCS: 221 bp and

SsCTR1: $461 \mathrm{bp)}$ were PCR-amplified from the cDNA library of S. sclerotiorum wild-type strain 1980 fragments were ligated into the plasmid vector pCIT (Yu et al, 2017) at the corresponding sites, and a hygromycin resistance gene cassette from pSKH (Hamid et al, 2013) was isolated and ligated into them, resulting the RNAi vectors, pSiatx, pSicox17, pSiccs and pSictr1. To generate S. sclerotiorum overexpression strains of these four genes, the full-length coding sequences were amplified by using the specific primers of overexpression vector construction in Table S1 and cloned into a hygromycin polyethylene glycol (PEG)-mediated transformation protocol (Rollins, 2003). 
Resource Center, Ohio State University, USA. The homozygous T-DNA insertion lines were confirmed with primers flanking the T-DNA insertions (Table EV3) and the left border primer LB1.3 (ATTTTGCCGATTTCGGAAC). To generate the overexpression lines, the coding sequences of these four genes were amplified from a cDNA library of $A$. thaliana leaves (Col [Columbia]-0). The amplicons were digested with $X b a \mathrm{I}$ and $\mathrm{XhoI}$ and ligated into the plant expression vector pBinGlyRed3, which contains a gene encoded red fluorescent protein (DsRed). The resulting vectors (pBinGlyRed-AtCCS, pBinGlyRed-AtCCH, pBinGlyRed-AtMT2A and pBinGlyRed-AtDRT112) were introduced into the Agrobacterium tumefaciens strain GV3101 by electroporation (Wise et al, 2006) and transformed into A. thaliana Col-0 by the floral dip method (Clough and Bent, 1998).

\section{RNA-seq and network analysis}

In our previous study (Ding et al, 2019), the stems of resistant and susceptible $B$. oleracea plants in a $F_{2}$ population which derived from the cross between a resistant B. oleracea genotype 'C01' (B. incana) and a susceptible $B$. oleracea genotype ' $\mathrm{C} 41$ ' (B. oleracea var. alboglabra) were inoculated with $S$. sclerotiorum, and lesions at 0,12 and 24 hpi were collected for transcriptome sequencing. To analyze the dynamic changes during infection, here we analyzed the DEGs of S. sclerotiorum and B. oleracea by using the DESeq package (Anders and Huber, 2010).

To reveal the pathways associated with 'copper ion homeostasis', the RNA of infected leaves was sequenced from A. thaliana wild type (Col-0), T-DNA mutants and overexpression lines during infection. Briefly, the detached leaves were inoculated with S. sclerotiorum wild-type strain 1980 (Mei et al, 2016), and RNA from the lesions was extracted with the RNAprep pure Plant Kit (DP 432, Tiangen Biotech (BEIJING) CO., LTD). The sequencing library was generated using the Illumina RNA Library Prep Kit (NEB, USA) following the manufacturer's recommendation, and sequenced on an Illumina HiSeq 4000 platform with three biological replicates. After removing low-quality reads and those with adapter 
347 sequences, poly- $\mathrm{N}$ sequence from the raw data, the clean reads were screened and aligned to the 348 reference genomes of $A$. thaliana (https://www.arabidopsis.org/download/index.jsp) and S. sclerotiorum 349 (http://fungidb.org/common/downloads/Current_Release/Ssclerotiorum1980UF-70/) by using the 350 TopHat program (http://ccb.jhu.edu/software/tophat/index.shtml) (Trapnell et al, 2009) with default 351 parameters except that the Q value was set to 100 . Gene expression was quantified using htseq-count 352 0.6.1p2 (https://htseq.readthedocs.io/). The raw counts were normalized by TMM normalization using 353 the edgeR package (Robinson et al, 2010) and the differential expression analysis was carried out using 354 the DESeq package (Anders and Huber, 2010).

The threshold determining the significance of DEGs among multiple tests was set at a false discovery rate $(F D R) \leq 0.001$ and $\mid \log _{2}$ ratio $\mid \geq 1$. GO (Gene Ontology) and KEGG (Kyoto Encyclopedia of Genes and Genomes) enrichment analyses were performed with an FDR $\leq 0.05$ as the threshold using AgriGO (Tian et al, 2017) and KOBAS 3.0 (http://kobas.cbi.pku.edu.cn/), respectively.

Weighted correlation networks were produced among the DEGs with R package WGCNA (Weighted Gene Co-expression Network Analysis) (Langfelder and Horvath, 2008). Networks were visualized by Cytoscape v3.4 (Shannon et al, 2003).

\section{Measurement of copper ion concentration}

Detached leaves and stems of B. napus cv. Zhongshuang 11, which was recognized as a moderately resistant rapeseed cultivar (Sun et al, 2017), were inoculated with S. sclerotiorum strains, and the uninfected $(\mathrm{U})$, margin $(\mathrm{M})$ and necrotic $(\mathrm{N})$ tissues were collected at $48 \mathrm{hpi}$. The tissues were washed with distilled water, dried for 1 week at $80^{\circ} \mathrm{C}$, and then washed with $11 \mathrm{~N} \mathrm{HNO}_{3}$. Copper concentration

367 of tissues was measured using atomic absorption spectroscopy (SPECTR AA220) at a wavelength of $368 \quad 324.8 \mathrm{~nm}$.

369 Quantitative RT-PCR 
Gene expression was analyzed by qRT-PCR using a Bio-Rad CFX96 Real Time System (Bio-Rad, USA) and QuantiTect SYBR Green PCR master mix (Bio-Rad, USA), according to the manufacturer's instructions. The SsTubulin and BoActin3 genes were used as the internal control for S. sclerotiorum and B. oleracea, respectively. All the qRT-PCR primers were listed in Table EV3. The PCR cycling conditions comprised 1 cycle of $95^{\circ} \mathrm{C}$ for $30 \mathrm{~s}$, then 39 cycles of $95^{\circ} \mathrm{C}$ for $5 \mathrm{~s}$ and $55-70^{\circ} \mathrm{C}$ for $1 \mathrm{~min}$, followed by a melting curve ramping from $65^{\circ} \mathrm{C}$ to $95^{\circ} \mathrm{C}$ with temperature increasing by $0.5^{\circ} \mathrm{C}$ every $5 \mathrm{~s}$ ( 1 cycle). Transcript levels were calculated from the threshold cycle using the $2^{-\Delta \Delta \mathrm{CT}}$ method (Livak and Schmittgen, 2001). Three replicates were performed for each gene and data were analyzed using CFX Manager ${ }^{\mathrm{TM}} \mathrm{v} 3.0$.

\section{Pathogenicity assays}

Pathogenicity of S. sclerotiorum was evaluated by infecting B. napus and A. thaliana according to the procedure described previously (Ding et al, 2019; Mei et al, 2016). The detached leaves at seedling stage and stems at flowering stage of B. napus, and in vivo leaves of 3-week-old A. thaliana, were inoculated with mycelium-colonized agar plugs $(0.6 \mathrm{~cm}$ for B. napus, $0.2 \mathrm{~cm}$ for A. thaliana) obtained from expanding margins of PDA-cultured colonies, with five replicates. The inoculation chamber was maintained at $85 \%$ relative humidity at $22^{\circ} \mathrm{C}$. The lesion size $\left(S, \mathrm{~cm}^{2}\right)$ for the leaves was calculated with the formula $S=\pi * a * b / 4$, where $a$ and $b$ represent the long and short diameter of an approximately elliptical lesion. The infection cushions on B. napus leaves at 6,9 and $12 \mathrm{hpi}$ were observed with a scanning electron microscope (JEOL JEM-6390LV).

\section{Antioxidant activity assays}

Superoxide $\left(\cdot \mathrm{O}_{2}^{-}\right)$accumulation was assayed by staining wild-type and transformant hyphae of $S$. sclerotiorum with NBT (Kumar et al, 2014). Hyphae on the PDA plate at 24 hpi were infiltrated under gentle vacuum with NBT staining solution for 5 hours and then washed 3 times with distilled water, 
prior to observation with a microscope. Meanwhile, expression of the S. sclerotiorum $\mathrm{Cu} / \mathrm{Zn}$ SOD gene (SsSOD1) was assayed by qRT-PCR, and the growth inhibition ratio was calculated by measuring the diameter every 12 hours when cultured on PDA in the presence of 2, 6, 10 and $15 \mathrm{mM} \mathrm{H}_{2} \mathrm{O}_{2}$. The enzyme activity of $\mathrm{Cu} / \mathrm{Zn}$ SOD of hyphae was tested using an enzyme activity kit (A001-1; Nanjing Jiancheng Bioengineering Institute) according to the manufacturer's protocol. (Kumar et al, 2014). Leaves were infiltrated under gentle vacuum with DAB or NBT staining solution for 5 hours. After staining, the staining solution was replaced with bleaching solution (ethanol: acetic acid: glycerol $=3: 1: 1)$. After $15 \pm 5$ min in a boiling water bath $\left(\sim 90-95^{\circ} \mathrm{C}\right)$, the bleaching solution was replaced with fresh bleaching solution and stained in $60 \%$ glycerin. Meanwhile, the enzyme activity of $\mathrm{Cu} / \mathrm{Zn}$ SOD was tested at 0, 6 and 12 hpi using an enzyme activity kit (A001-1; Nanjing Jiancheng Bioengineering Institute) according to the manufacturer's protocol.

\section{Oxalic acid assays}

406 Five agar plugs $(6 \mathrm{~mm})$ from the advancing edge of each $S$. sclerotiorum strain were transferred into 5 $\mathrm{ml}$ PDA medium with $50 \mathrm{mg} / \mathrm{L}$ bromophenol blue and incubated at $20^{\circ} \mathrm{C}$ with shaking at $150 \mathrm{rpm}$ for 2 days. Prior to assaying the concentration of OA in the solution, a standard curve was generated using OA standard samples with a spectrometer (Evolution ${ }^{\mathrm{TM}}$ 201/220, ThermoFisher, USA). Meanwhile, the

$\mathrm{pH}$ value of the solution was measured with a $\mathrm{pH}$ meter (INESA, China), and the expression of $S s O A H$ was assayed in S. sclerotiorum strains by qRT-PCR.

Acknowledgements This study was financially supported by the National Natural Science Foundation of China (31671726, 31801395 and 31971978), the Natural Science Foundation of Chongqing (cstc2017shms-Xdny80050, cstc2019jcyj-zdxmX0012 and cstc2019jcyj-msxm2511) and Fundamental 
Research Funds for the Central Universities (XDJK2018AA004 and XDJK2018B022). We sincerely acknowledge Dr. Pradeep Kachroo and Dr. Zhonglin Mou for critical comments.

\section{Author contributions}

W.Q. designed the experiments, Y.D., J.M. performed experiments, analyzed the data, and wrote the manuscript. Y.C. performed and analyzed the RNA sequencing experiments. W.Y. performed cloning and transformation experiments. Y.M. performed copper ion concentration measurement and oxalic acid assays. B.Y., Y.Y. and K.R. performed the pathogenicity and antioxidant activity analysis. J.O.D and J. L. analyzed the data and helped write the manuscript. All authors reviewed the manuscript before publication.

\section{Conflict of interest}

The authors declare that they have no conflict of interest.

\section{References}

Abdel-Ghany SE (2009) Contribution of plastocyanin isoforms to photosynthesis and copper homeostasis in Arabidopsis thaliana grown at different copper regimes. Planta 229: 767-779

Amselem J, Cuomo CA, van Kan JA, Viaud M, Benito EP, Couloux A, Coutinho PM, de Vries RP, Dyer PS, Fillinger S et al ( 2011) Genomic analysis of the necrotrophic fungal pathogens Sclerotinia sclerotiorum and Botrytis cinerea. PLoS Genet 7: e1002230

Anders S, Huber W (2010) Differential expression analysis for sequence count data. Genome Biol 11

Arnesano F, Balatri E, Banci L, Bertini I, Winge DR (2005) Folding studies of Cox17 reveal an important interplay of cysteine oxidation and copper binding. Structure 13: 713-722

Banci L, Bertini I, Cantini F, Kozyreva T, Massagni C, Palumaa P, Rubino JT, Zovo K (2012) Human superoxide dismutase 1 (hSOD1) maturation through interaction with human copper chaperone for 
SOD1 (hCCS). Proc Natl Acad Sci U S A 109: 13555-13560

442

443

444

445

446

447

448

449

450

451

452

453

454

455

456

457

458

459

460

461

462

463

Berterame NM, Martani F, Porro D, Branduardi P (2018) Copper homeostasis as a target to improve Saccharomyces cerevisiae tolerance to oxidative stress. Metab Eng 46: 43-50

Besold AN, Culbertson EM, Culotta VC (2016) The Yin and Yang of copper during infection. J Biol Inorg Chem 21: 137-144

Cankorur-Cetinkaya A, Eraslan S, Kirdar B (2016) Transcriptomic response of yeast cells to ATX1 deletion under different copper levels. BMC Genomics 17: 489

Carr HS, Winge DR (2003) Assembly of cytochrome c oxidase within the mitochondrion. Acc Chem Res 36: 309-316

Chu CC, Lee WC, Guo WY, Pan SM, Chen LJ, Li HM, Jinn TL (2005) A copper chaperone for superoxide dismutase that confers three types of copper/zinc superoxide dismutase activity in Arabidopsis. Plant Physiol 139: 425-436

Clough SJ, Bent AF (1998) Floral dip: a simplified method for Agrobacterium-mediated transformation of Arabidopsis thaliana. Plant J 16: 735-743

Cobine PA, Pierrel F, Winge DR (2006) Copper trafficking to the mitochondrion and assembly of copper metalloenzymes. Biochim Biophys Acta 1763: 759-772

Crawford A, Wilson D (2015) Essential metals at the hos-pathogen interface: nutritional immunity and micronutrient assimilation by human fungal pathogens. FEMS Yeast Res 15: 7

Culotta VC, Yang M, O'Halloran TV (2006) Activation of superoxide dismutases: putting the metal to the pedal. Biochim Biophy Acta 1763: 747-758

del Pozo T, Cambiazo V, González M (2010) Gene expression profiling analysis of copper homeostasis in Arabidopsis thaliana. Biochem Biophys Res Commun 393: 248-252

Ding C, Yin J, Tovar EM, Fitzpatrick DA, Higgins DG, Thiele DJ (2011) The copper regulon of the 

human fungal pathogen Cryptococcus neoformans H99. Mol Microbiol 81: 1560-1576

Ding Y, Mei J, Chai Y, Yu Y, Shao C, Wu Q, Disi JO, Li Y, Wan H, Qian W (2019) Simultaneous transcriptome analysis of host and pathogen highlights the interaction between Brassica oleracea and Sclerotinia sclerotiorum. Phytopathology 109: 542-550

Douglas LM, Wang HX, Keppler-Ross S, Dean N, Konopka JB (2012) Sur7 promotes plasma membrane organization and is needed for resistance to stressful conditions and to the invasive growth and virulence of Candida albicans. MBio 3: e00254-11

Foyer CH, Noctor G (2013) Redox signaling in plants. Antioxid Redox Signal 18: 2087-2090

Garg H, Li H, Sivasithamparam K, Barbetti MJ (2013) Differentially expressed proteins and associated histological and disease progression changes in cotyledon tissue of a resistant and susceptible genotype of Brassica napus infected with Sclerotinia sclerotiorum. PLoS one 8: e65205

Garg H, Li H, Sivasithamparam K, Kuo J, Barbetti MJ (2010) The infection processes of Sclerotinia sclerotiorum in cotyledon tissue of a resistant and a susceptible genotype of Brassica napus. Ann Bot 106: 897-908

Gleason JE, Li CX, Odeh HM, Culotta VC (2014) Species-specific activation of Cu/Zn SOD by its CCS copper chaperone in the pathogenic yeast Candida albicans. J Biol Inorg Chem 19: 595-603

Govrin EM, Levine A (2000) The hypersensitive response facilitates plant infection by the necrotrophic pathogen Botrytis cinerea. Curr Biol 10: 751-757

Gu CS, Liu LQ, Deng YM, Zhu XD, Huang SZ, Lu XQ (2015) The heterologous expression of the Iris lactea var. chinensis type 2 metallothionein IlMT2b gene enhances copper tolerance in Arabidopsis thaliana. Bull Environ Contam Toxicol 94: 247-253

Hamid MI, Zeng F, Cheng J, Jiang D, Fu Y (2013) Disruption of heat shock factor 1 reduces the formation of conidia and thermotolerance in the mycoparasitic fungus Coniothyrium minitans. 
Heller J, Tudzynski P (2011) Reactive oxygen species in phytopathogenic fungi: signaling, development, and disease. Anпu Rev Phytopathol 49: 369-390

490

491

492

493

494

495

496

497

498

499

500

501

502

503

504

505

506

507

508

509

Himelblau E, Mira H, Lin SJ, Culotta VC, Peñarrubia L, Amasino RM (1997) Identification of a functional homolog of the yeast copper homeostasis gene ATX1 from Arabidopsis. Plant Physiol 117: $1227-1234$

Hood MI, Skaar EP (2012) Nutritional immunity: transition metals at the pathogen-host interface. Nat Rev Microbiol 10: 525-537

Jones JDG, Dangl JL (2006) The plant immune system. Nature 444: 323-329

Kim H, Chen C, Kabbage M, Dickman MB (2011) Identification and Characterization of Sclerotinia sclerotiorum NADPH oxidases. Appl Environ Microb 77: 7721-7729

Kim KS, Min JY, Dickman MB (2008) Oxalic acid is an elicitor of plant programmed cell death during Sclerotinia sclerotiorum disease development. Mol Plant Microbe In 21: 605-612

Kim YO, Kang H (2018) Comparative expression analysis of genes encoding metallothioneins in response to heavy metals and abiotic stresses in rice (Oryza sativa) and Arabidopsis thaliana. Biosci Biotechnol Biochem 82: 1656-1665

Kumar D, Yusuf MA, Singh P, Sardar M, Sarin NB (2014) Histochemical detection of superoxide and $\mathrm{H}_{2} \mathrm{O}_{2}$ accumulation in Brassica juncea seedlings. Bio-Protocol 4: e1108

Ladomersky E, Petris MJ (2015) Copper tolerance and virulence in bacteria. Metallomics 7: 957-964

Langfelder P, Horvath S (2008) WGCNA: an R package for weighted correlation network analysis. BMC Bioinformatics 9: 559

Li CX, Gleason JE, Zhang SX, Bruno VM, Cormack BP, Culotta VC (2015) Candida albicans adapts to host copper during infection by swapping metal cofactors for superoxide dismutase. Proc Natl Acad 
Sci U S A 112: 5336-5342

Li J, Zhang Y, Zhang Y, Yu PL, Pan H, Rollins JA (2018) Introduction of large sequence inserts by CRISPR-Cas9 to create pathogenicity mutants in the multinucleate filamentous pathogen Sclerotinia sclerotiorum. MBio 9: e00567-18

Liang X, Rollins JA (2018) Mechanisms of broad host range necrotrophic pathogenesis in Sclerotinia sclerotiorum. Phytopathology 108: 1128-1140

Liang Y, Srivastava S, Rahman MH, Strelkov SE, Kav NN (2008) Proteome changes in leaves of Brassica napus L. as a result of Sclerotinia Sclerotiorum challenge. J Agric Food Chem 56: 1963-1976

Liberti D, Rollins J, Dobinson K (2013) Peroxysomal carnitine acetyl transferase influences host colonization capacity in Sclerotinia sclerotiorum. Mol Plant Microbe In 26: 768-780

Lin SJ, Pufahl RA, Dancis A, O’Halloran TV, Culotta VC (1997) A role for the Saccharomyces cerevisiae ATX1 gene in copper trafficking and iron transport. J Biol Chem 272: 9215-9220

Liu Y, He C (2017) A review of redox signaling and the control of MAP kinase pathway in plants. Redox boil 11: 192-204

Livak KJ, Schmittgen TD (2001) Analysis of relative gene expression data using real-time quantitative PCR and the $2^{-\Delta \Delta C T}$ method. Methods 25: 402-408

Lu H, Higgins VJ (1999) The effect of hydrogen peroxide on the viability of tomato cells and of the fungal pathogen Cladosporium fulvum. Physiol Mol Plant Pathol 54: 131-143

Malavia D, Crawford A, Wilson D (2017) Nutritional immunity and fungal pathogenesis: the struggle for micronutrients at the host-pathogen interface. Adv Microb Physiol 70: 85-103

Marschall R, Tudzynski P (2014) A new and reliable method for live imaging and quantification of reactive oxygen species in Botrytis cinerea: Technological advancement. Fungal Genet Biol 71: 


\section{8-75}

Martins F, Pereira JA, Baptista P (2014) Oxidative stress response of Beauveria bassiana to Bordeaux mixture and its influence on fungus growth and development. Pest Manag Sci 70: 1220-1227

Mei J, Ding Y, Li Y, Tong C, Du H, Yu Y, Wan H, Xiong Q, Yu J, Liu S et al (2016) Transcriptomic comparison between Brassica oleracea and rice (Oryza sativa) reveals diverse modulations on cell death in response to Sclerotinia sclerotiorum. Sci Rep 10: 34900

Mignolet-Spruyt L, Xu E, Idänheimo N, Hoeberichts FA, Mühlenbock P, Brosché M, Van BF, Kangasjärvi J (2016) Spreading the news: subcellular and organellar reactive oxygen species production and signalling. J Exp Bot 67: 3831-3844

Mittler R (2017) ROS are good. Trends Plant Sci 22: 11-19

Mittler R, Vanderauwera S, Suzuki N, Miller G, Tognetti VB, Vandepoele K, Gollery M, Shulaev V, Van BF (2011) ROS signaling: the new wave? Trends Plant Sci 16: 300-309

Pena MM, Puig S, Thiele DJ (2000) Characterization of the Saccharomyces cerevisiae high affinity copper transporter Ctr3. J Biol Chem 275: 33244-33251

Pilon M, Abdel-Ghany SE, Cohu CM, Gogolin KA, Ye H (2006) Copper cofactor delivery in plant cells. Curr. Opin. Plant Biol 9: 256-263

Poyton RO, Goehring B, Droste M, Sevarino KA, Allen LA, Zhao J (1995) Cytochrome-c oxidase from Saccharomyces cerevisiae. Methods Enzymol 260: 97-116

Rae TD, Schmidt PJ, Pufahl RA, Culotta VC, O'Halloran TV (1999) Undetectable intracellular free copper: The Requirement of a copper chaperone for superoxide dismutase. Science 284: 805-808

Ranjan A, Jayaraman D, Grau C, Hill JH, Whitham SA, Ané JM, Smith DL, Kabbage M (2018) The pathogenic development of Sclerotinia sclerotiorum in soybean requires specific host NADPH oxidases. Mol Plant Pathol 19: 700-714 
Rees EM, Lee J, Thiele DJ (2004) Mobilization of intracellular copper stores by the ctr2 vacuolar copper transporter. J Biol Chem 279: 54221-54229

Robinson MD, McCarthy DJ, Smyth GK (2010) edgeR: a Bioconductor package for differential expression analysis of digital gene expression data. Bioinformatics 26: 139-140

Rollins JA (2003) The Sclerotinia sclerotiorum pacl gene is required for sclerotial development and virulence. Mol Plant Microbe In 16: 785-795

Samanovic MI, Ding C, Thiele DJ, Darwin KH (2012) Copper in microbial pathogenesis: meddling with the metal. Cell Host Microbe 11:106-115

Shannon P, Markiel A, Ozier O, Baliga NS, Wang JT, Ramage D, Amin N, Schwikowski B, Ideker T (2003) Cytoscape: a software environment for integrated models of biomolecular interaction networks. Genome Res 13: 2498-504

Sun F, Fan G, Hu Q, Zhou Y, Guan M, Tong C, Li J, Du D, Qi C, Jiang L et al (2017) The high-quality genome of Brassica napus cultivar 'ZS11' reveals the introgression history in semi-winter morphotype. Plant J 92: 452-468

Tian T, Liu Y, Yan H, You Q, Yi X, Du Z, Xu W, Su, Z (2017) agriGO v2.0: a GO analysis toolkit for the agricultural community. Nucleic Acids Res 45: 122-129

Trapnell C, Pachter L, Salzberg SL (2009) TopHat: discovering splice junctions with RNA-Seq. Bioinformatics

Veluchamy S, Williams B, Kim K, Dickman M (2012) The CuZn superoxide dismutase from Sclerotinia sclerotiorum is involved with oxidative stress tolerance, virulence, and oxalate production. Physiol Mol Plant Pathol 78:14e23

Wang Z, Fang H, Chen Y, Chen K, Li G, Gu S, Tan X (2014) Overexpression of BnWRKY33 in oilseed rape enhances resistance to Sclerotinia sclerotiorum. Mol Plant Pathol 15: 677-689 
Wei L, Jian H, Lu K, Filardo F, Yin N, Liu L, Qu C, Li W, Du H, Li, J (2016) Genome-wide association analysis and differential expression analysis of resistance to Sclerotinia stem rot in Brassica napus. Plant Biotechnol J 14: 1368-1380

Weissman Z, Berdicevsky I, Cavari BZ, Kornitzer D (2000) The high copper tolerance of Candida albicans is mediated by a P-type ATPase. Proc Natl Acad Sci U S A 97: 3520-3525

Wen L, Tan TL, Shu JB, Chen Y, Liu Y, Yang ZF, Zhang QP, Yin M, Tao J, Guan CY (2013) Using proteomic analysis to find the proteins involved in resistance against Sclerotinia sclerotiorum in adult Brassica napus. Eur J Plant Pathol 137: 505-523

Wise AA, Liu Z, Binns AN (2006) Three methods for the introduction of foreign DNA into Agrobacterium. Methods Mol Biol 343: 43-53

Yang B, Srivastava S, Deyholos MK, Kav NNV (2007) Transcriptional profiling of canola (Brassica napus L.) responses to the fungal pathogen Sclerotinia sclerotiorum. Plant Sci 173: 156-171

Yruela I (2009) Copper in plants: acquisition, transport and interactions. Funct Plant Biol 39: 409-430

Yu Y, Xiao J, Zhu W, Yang Y, Mei J, Bi C, Qian W, Qing L, Tan W (2017) Ss-Rhsl, a secretory Rhs repeat-containing protein, is required for the virulence of Sclerotinia sclerotiorum. Mol Plant Pathol 18: 1052-1061

\section{Figure legends}

Figure 1. Copper is involved in the interaction between Brassica and Sclerotinia sclerotiorum.

A Gene Ontology (GO) biological processes represented by S. sclerotiorum DEGs (differentially expressed genes; 24 hours post inoculation [hpi] vs 12 hpi) in infected B. oleracea stems.

B GO biological processes represented by resistant and susceptible B. oleracea DEGs (24 hpi vs 12 hpi). 
601 C Copper concentration in uninfected (U), margin (M) and necrotic (N) tissues of B. napus leaves and 602 stems at 48 hpi. Error bars indicate the standard deviation of three replicates. *: represents significant 603 difference compared to the mock value at 0.05 level, NS indicates no significant difference at 0.05 604 level (Student's $t$-test). Ss R24/R12: S. sclerotiorum DEGs in resistant B. oleracea identified by 605 comparing 24 hpi to 12 hpi; Ss S24/S12: S. sclerotiorum DEGs in susceptible B. oleracea identified 606 by comparing 24 hpi to 12 hpi; Bol R24/R12: B. oleracea DEGs in resistant B. oleracea identified by 607 comparing 24 hpi to $12 \mathrm{hpi}$; Bol S24/S12: B. oleracea DEGs in susceptible $B$. oleracea identified by 608 comparing 24 hpi to 12 hpi.

609

610

611

612

\section{Figure 2. Copper ion homeostasis genes promote resistance to $S$. sclerotiorum in Arabidopsis.}

A Disease symptoms in Arabidopsis wild type (WT), T-DNA mutants (atccs, atmt2a, atdrt112 and atcch) and overexpression lines (OX-AtCCS, OX-AtMT2A, OX-AtDRT112 and OX-AtCCH) corresponding to copper ion homeostasis genes at 4 days post inoculation (dpi) of S. sclerotiorum wild-type strain 1980.

B Lesion size at 24 hpi in A. Error bars indicate the standard deviation of six replicates. *: represents significant difference from WT at 0.05 level (Student's $t$-test).

Figure 3. The biological process of 'copper ion homeostasis' is associated with 'response to oxidative stress' in the host.

A GO terms (overlapped among the four genes) significantly enriched among the up-regulated DEGs at 12 hpi between Arabidopsis overexpression (OX) lines and wild type (WT) and between OX and T-DNA mutants.

B GO terms significantly enriched among 394 DEGs in the module that significantly and negatively correlated with lesion size based on WGCNA (Weighted Gene Co-expression Network Analysis). The network was visualized using Cytoscape v3.4. 


\section{Figure 4. Copper ion homeostasis genes promote antioxidant activity in host.}

Antioxidant activity assays in wild type (WT), overexpression lines (OX) and T-DNA mutants of copper ion homeostasis genes in Arabidopsis at 0, 6 and $12 \mathrm{hpi}$.

A DAB $\left(\mathrm{H}_{2} \mathrm{O}_{2}\right.$ accumulation $)$ and NBT $\left(\cdot \mathrm{O}_{2}{ }^{-}\right.$accumulation $)$staining. One representative replicate from the five experiments is shown. The red arrows indicate the inoculant columns.

B Enzyme activity of $\mathrm{Cu} / \mathrm{Zn}$ SOD in leaves of Arabidopsis at 0, 6 and 12 hpi. Error bars indicate standard deviation for three replicates.

Figure 5. SsCTR1, SsCCS and SsATX1 associated with copper absorption during infection.

A Relative expression of SSCTR1, SsCCS and SSATX1 in wild-type strain 1980, silenced and overexpressing strains as indicated by qRT-PCR analysis. The quantity of S. sclerotiorum Tubulin cDNA was used to normalize different samples. Error bars indicate the standard deviation of three independent samples.

B Copper concentration in uninfected (U), margin $(\mathrm{M})$ and necrotic $(\mathrm{N})$ tissues of B. napus leaves at 48 hpi with wild-type, overexpressing and silenced strains. Error bars indicate the standard deviation of three replicates. *: represents significant difference between the transgenic strains and wild-type strain at the level of 0.05 . NS indicates no significant difference at 0.05 level (Student's $t$-test).

Figure 6. Trace copper restores the virulence of silenced strains of S. sclerotiorum.

A Disease symptoms of leaves after inoculation with S. sclerotiorum wild-type strain 1980, overexpressing strains (OX, OXccs, OXatx1, OXcox17 and OXctr1) and silenced strains (Si, Siccs, Siatx1, Sicox17 and Sictr1) for genes involved in 'copper ion transport/import'. The tests of the silenced strains were supplemented with low concentrations of $\mathrm{CuSO}_{4}$ solution, while the tests of 1980 and the overexpressing strains were sprayed with water. One representative replicate from the five experiments is shown. 
647 B Quantitation of lesion sizes at 48 hpi produced by wild-type, overexpressing and silenced strains when 648 supplemented with low and high concentrations of $\mathrm{CuSO}_{4}$ solution. Error bars indicate standard 649 deviation of five replicates. NS: represents no significant difference from wild-type strain at 0.05 level 650 (Student's $t$-test).

651 C The growth on PDA for wild-type, overexpressing and silenced strains with supplementing low and 652 high concentration of $\mathrm{CuSO}_{4}$. Error bars indicate standard deviation of five replicates. *: represents 653 significant difference between the transgenic strains and wild-type strain at the level of 0.05 . NS: 654 represents no significant difference from wild-type strain at 0.05 level (Student's $t$-test).

655 Figure 7. SsCCS promotes antioxidant activity in S. sclerotiorum.

656 A Accumulation of $\cdot \mathrm{O}_{2}^{-}$(NBT staining at $24 \mathrm{hpi}$ ) in the hyphae tips and growth phenotypes on PDA 657 supplemented with different concentrations of $\mathrm{H}_{2} \mathrm{O}_{2}$ at 4 dpi. One representative replicate from the 658 five experiments is shown.

659 B The inhibition rate of hyphal growth on PDA supplemented with different concentrations of $\mathrm{H}_{2} \mathrm{O}_{2}$. 660 Error bars indicate standard deviation of five replicates. *: represents significant difference between 661 the transgenic strains and wild-type strain at the level of 0.05. NS: represents no significant difference 662 from wild-type strain at 0.05 level (Student's $t$-test).

663 C Relative expression of S. sclerotiorum SSSOD1 in wild-type strain 1980, silenced and overexpressing 664 strains as indicated by qRT-PCR analysis. The quantity of S. sclerotiorum Tubulin cDNA was used to 665 normalize different samples. Error bars indicate the standard deviation of three independent samples. $666 *$ : represents significant difference between the transgenic strains and wild-type strain at the level of $667 \quad 0.05$ (Student's $t$-test). 
668 D Enzyme activity of Cu/Zn SOD in hyphae of wild-type, silenced and overexpressing strains. Error

669 bars indicate the standard deviation of three independent samples. *: represents significant difference

670 between the transgenic strains and wild-type strain at the level of 0.05 (Student's $t$-test).

\section{Figure 8. Model depicting the battle for copper acquisition between host and S. sclerotiorum}

672 during infection.

673 S. sclerotiorum promotes its copper uptake systems which hijack host-derived copper, and activates its

674 ROS scavenging system for survival and virulence, while a resistant host induces 'nutritional immunity'

675 that restricts the supply of essential copper nutrients to S. sclerotiorum by maintaining 'copper ion

676 homeostasis'. 12/0: gene expression comparison of 12 hpi to 0 hpi in host, 24/12: gene expression

677 comparison of 24 hpi to 12 hpi in host.

678 


\section{Expanded View Figure legends}

Figure EV1. DEG (differentially expressed gene) analysis of Sclerotinia sclerotiorum and Brassica oleracea (24 hours post inoculation [hpi] vs 12 hpi).

A DEGs of S. sclerotiorum during infection in the resistant (R-Ss) and susceptible (S-Ss) B. oleracea.

B DEGs of resistant (R-Bol) and susceptible (S-Bol) B. oleracea.

C Heat map of S. sclerotiorum DEGs involved in the process 'copper ion import' and 'copper ion transport'.

D Heat map of B. oleracea DEGs involved in the process 'copper ion homeostasis'. Ss R24/R12: the $S$. sclerotiorum DEGs in resistant B. oleracea by comparing 24 hpi to 12 hpi; Ss S24/S12: the $S$. sclerotiorum DEGs in susceptible $B$. oleracea by comparing 24 hpi to 12 hpi; Bol R24/R12: the $B$. oleracea DEGs in resistant B. oleracea by comparing 24 hpi to $12 \mathrm{hpi}$; Bol S24/S12: the B. oleracea DEGs in susceptible B. oleracea by comparing 24 hpi to $12 \mathrm{hpi}$.

Figure EV2. DEGs and DEG network analysis of Arabidopsis and S. sclerotiorum during infection.

A Relative expression level of target genes in the Arabidopsis T-DNA mutants and overexpression lines (OX) in comparison with the wild type Col-0 as revealed by the RNA-seq.

B Weighted Gene Co-expression Network Analysis (WGCNA) of the DEGs between OX lines and T-DNA mutants in Arabidopsis.

C WGCNA of S. sclerotiorum DEGs during infection of Arabidopsis overexpression lines and T-DNA mutants.

Figure EV3. Copper ion homeostasis-related genes in Arabidopsis are associated with resistance to Botrytis cinerea.

A Disease symptoms at 24 hpi in Arabidopsis wild type (WT), T-DNA mutants (atccs, atmt2a, atdrt112 and atcch) and overexpression lines (OX-AtCCS, OX-AtMT2A, OX-AtDRT112 and OX-AtCCH) 
after inoculation of B. cinerea strain B05.10.

703

704

705

706

707

708

709

710

711

712

713

714

715

716

717

718

B Lesion size in (A). Error bars indicate the standard deviation of six replicates. *: represents significant difference of WT with T-DNA mutants and overexpression lines of Arabidopsis at the level of 0.05 (Student's $t$-test).

Figure EV4. Virulence assays of the wild-type, silenced and overexpressing strains of $S$. sclerotiorum.

A The silenced (RNAi: RNA interference) and overexpressed (OX) vectors.

B Relative expression level of the target genes in silenced, overexpressed and wild type strain 1980 on PDA medium as determined by qRT-PCR. The quantity of S. sclerotiorum Tubulin cDNA normalized different samples. Error bars indicate the standard deviation of three independent samples. *: represents significant difference between the transgenic strains and wild-type strain at the level of 0.05 (Student's $t$-test).

C Virulence on Brassica napus Zhongshuang 11 in detached leaves at 48 hpi. Error bars indicate the standard deviation of six replicates. *: represents significant difference between the transgenic strains and wild-type strain at the level of 0.05 (Student's $t$-test).

D Virulence in Arabidopsis thaliana plants at 24 hpi. Error bars indicate the standard deviation of six replicates. *: represents significant difference between the transgenic strains and wild-type strain at the level of 0.05 (Student's $t$-test).

E Disease symptoms at 4 dpi (days-post inoculation) in A. thaliana plants.

Figure EV5. The expression disruption of the copper ion transport/import genes suppresses the infection cushion formation and OA production in S. sclerotiorum.

A Infection cushion observation on B. napus leaves inoculated with the wild-type, silenced and overexpressing strains at 6,9 and $12 \mathrm{hpi}$. Bar $=100 \mu \mathrm{m}$. Experiments were repeated with three times 
725 with similar results.

726 B OA production of S. sclerotiorum strains. Error bars indicate the standard deviation of three replicates.

$727 *$ : represents significant difference between the transgenic strains and wild-type strain at the level of $728 \quad 0.05$ (Student's $t$-test).

729 C Ambient $\mathrm{pH}$ of strains in PDA.

730 D The relative expression of $S$. sclerotiorum SsOAH among S. sclerotiorum strains. The quantity of $S$. 731 sclerotiorum Tubulin cDNA normalized different samples. Error bars indicate the standard deviation 732 of three independent samples. *: represents significant difference between the transgenic strains and 733 wild-type strain at the level of 0.05 (Student's $t$-test).

735 Expanded View Table legends

736 Table EV1. 7321 Arabidopsis DEGs between the overexpression lines and T-DNA mutants.

737 Table EV2. 1273 S. sclerotiorum DEGs during infecting Arabidopsis overexpression lines and T-DNA 738 mutants.

739 Table EV3. Primers used in this study. 
$\mathbf{A}$

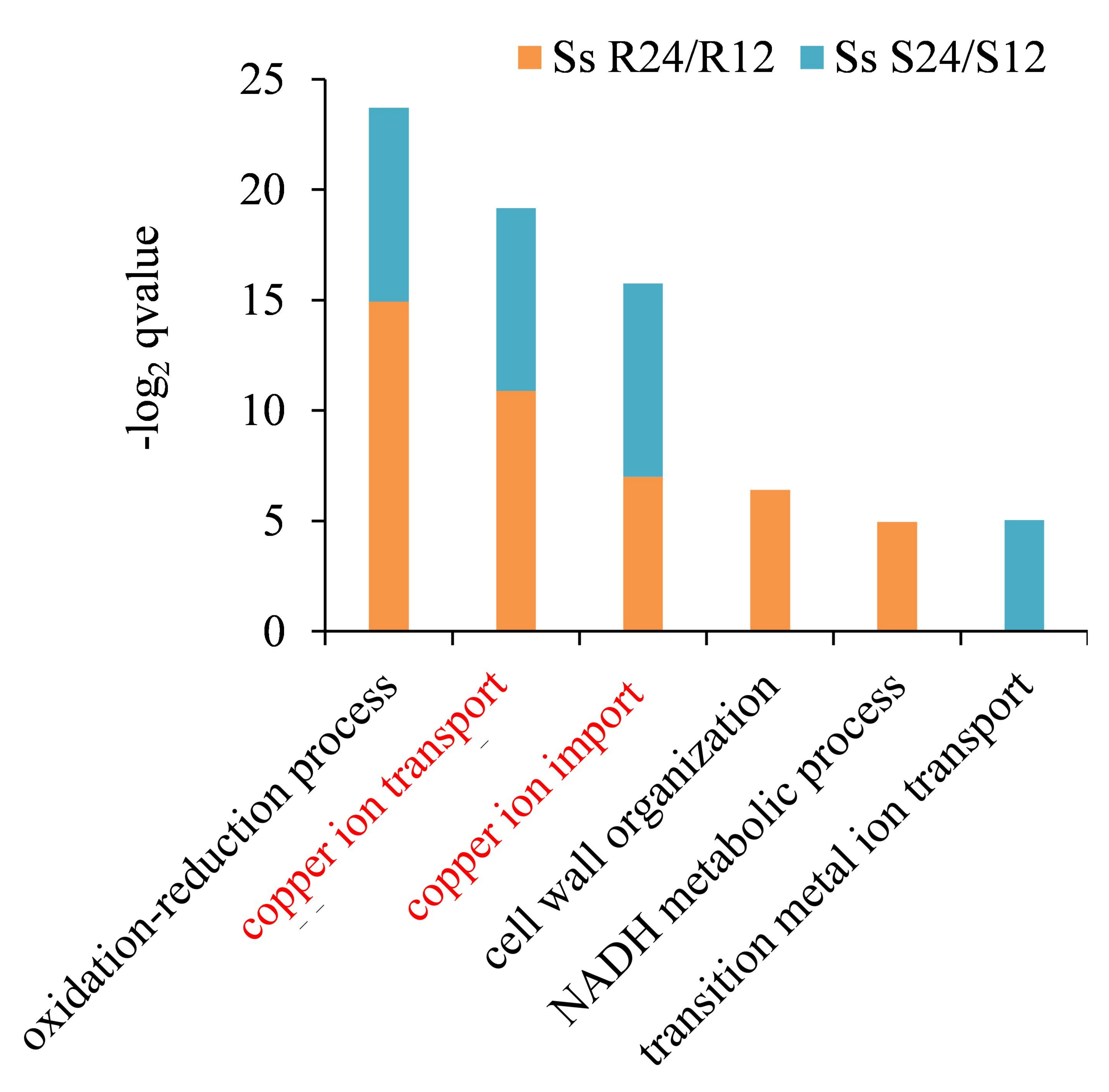

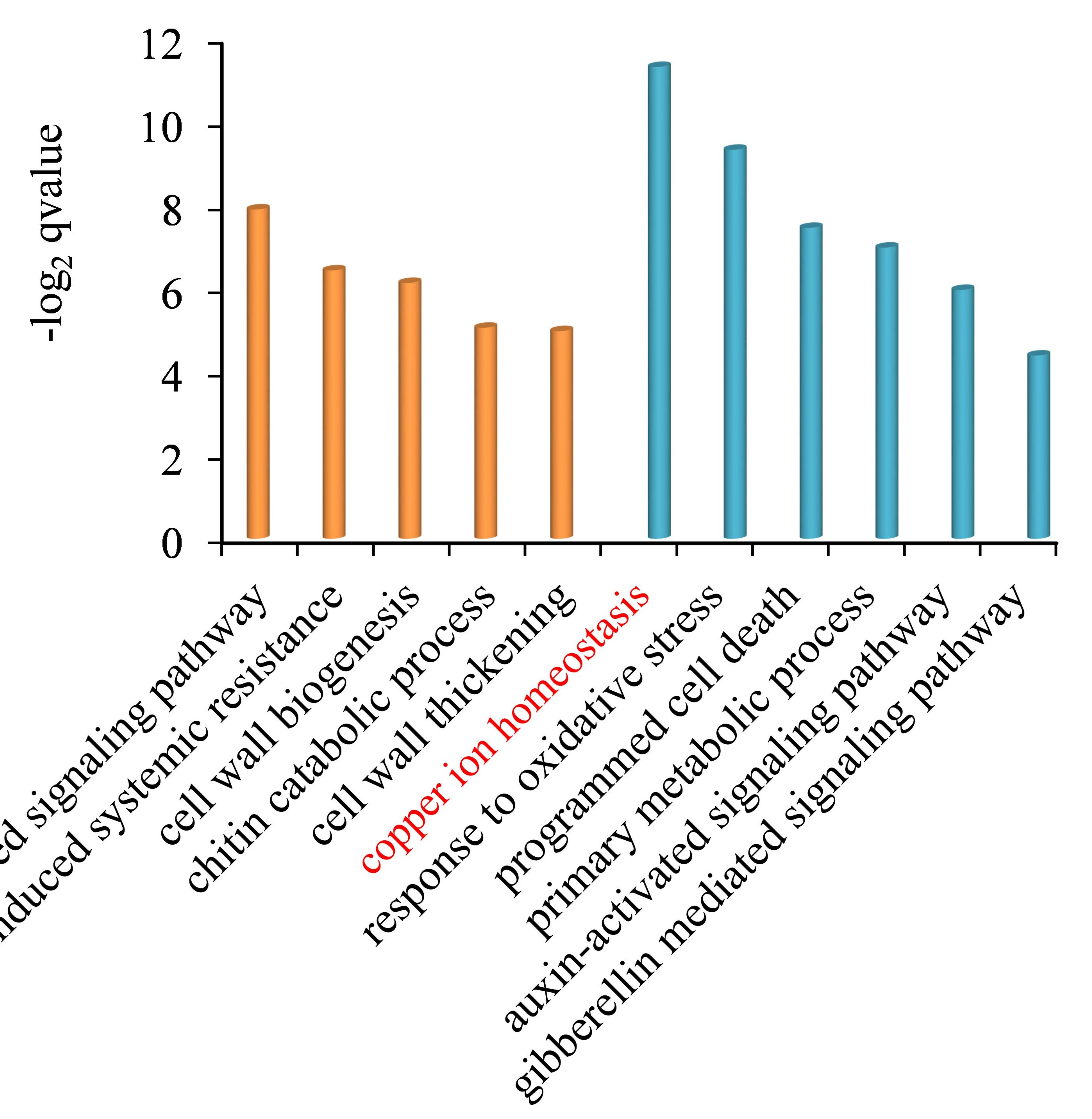

C

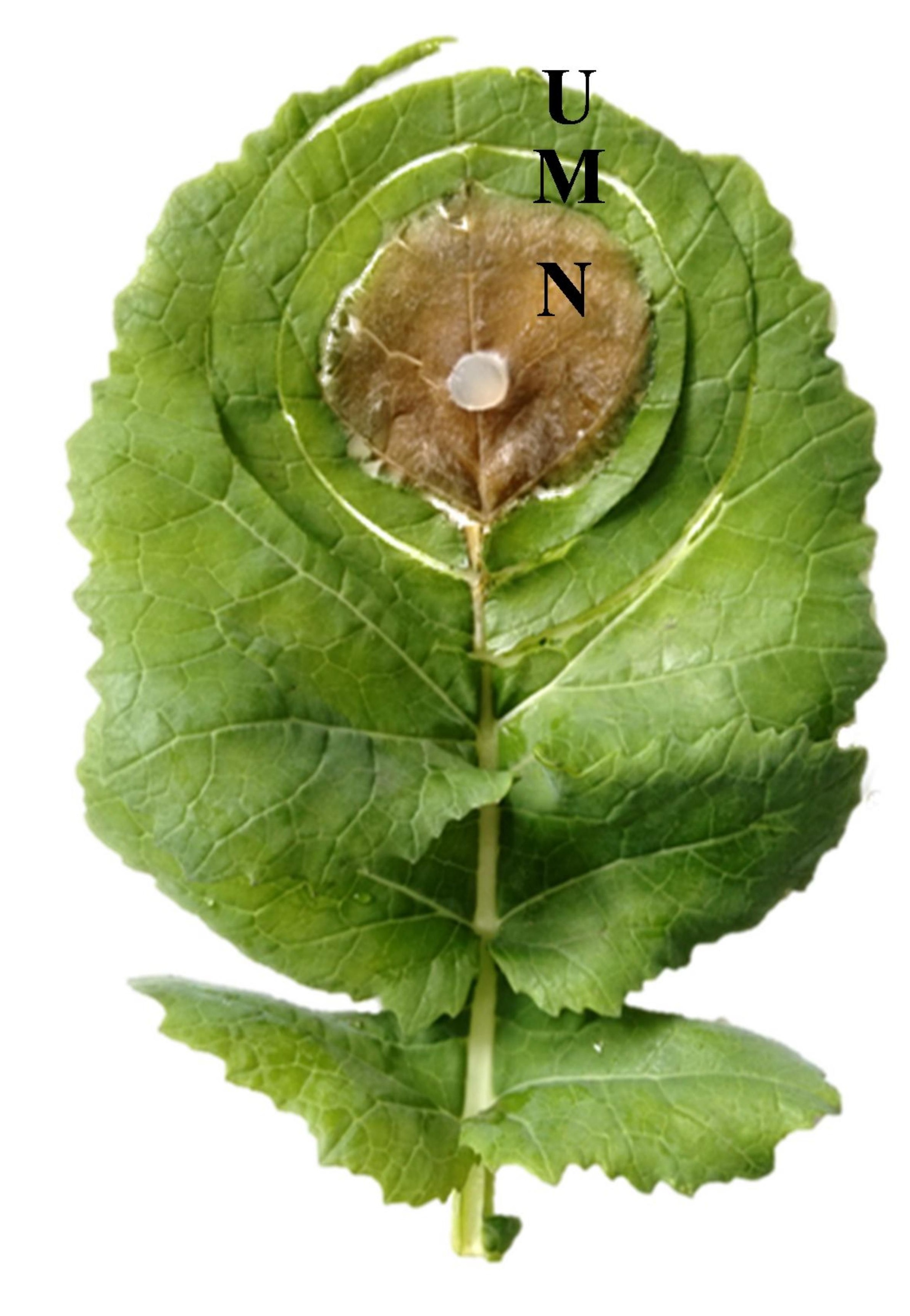

$\mathrm{Cu}$ in leaf
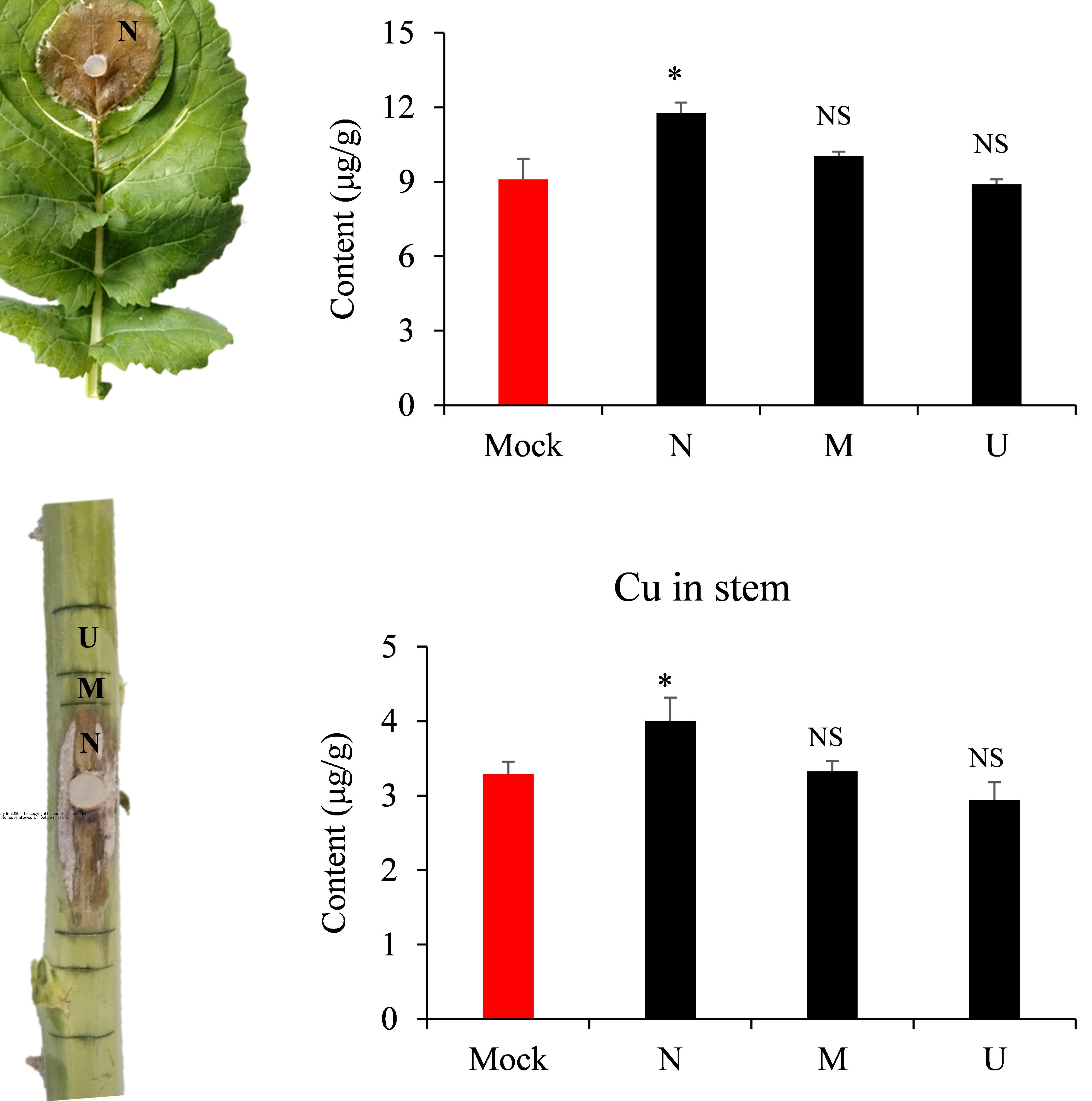

$\mathrm{Cu}$ in stem

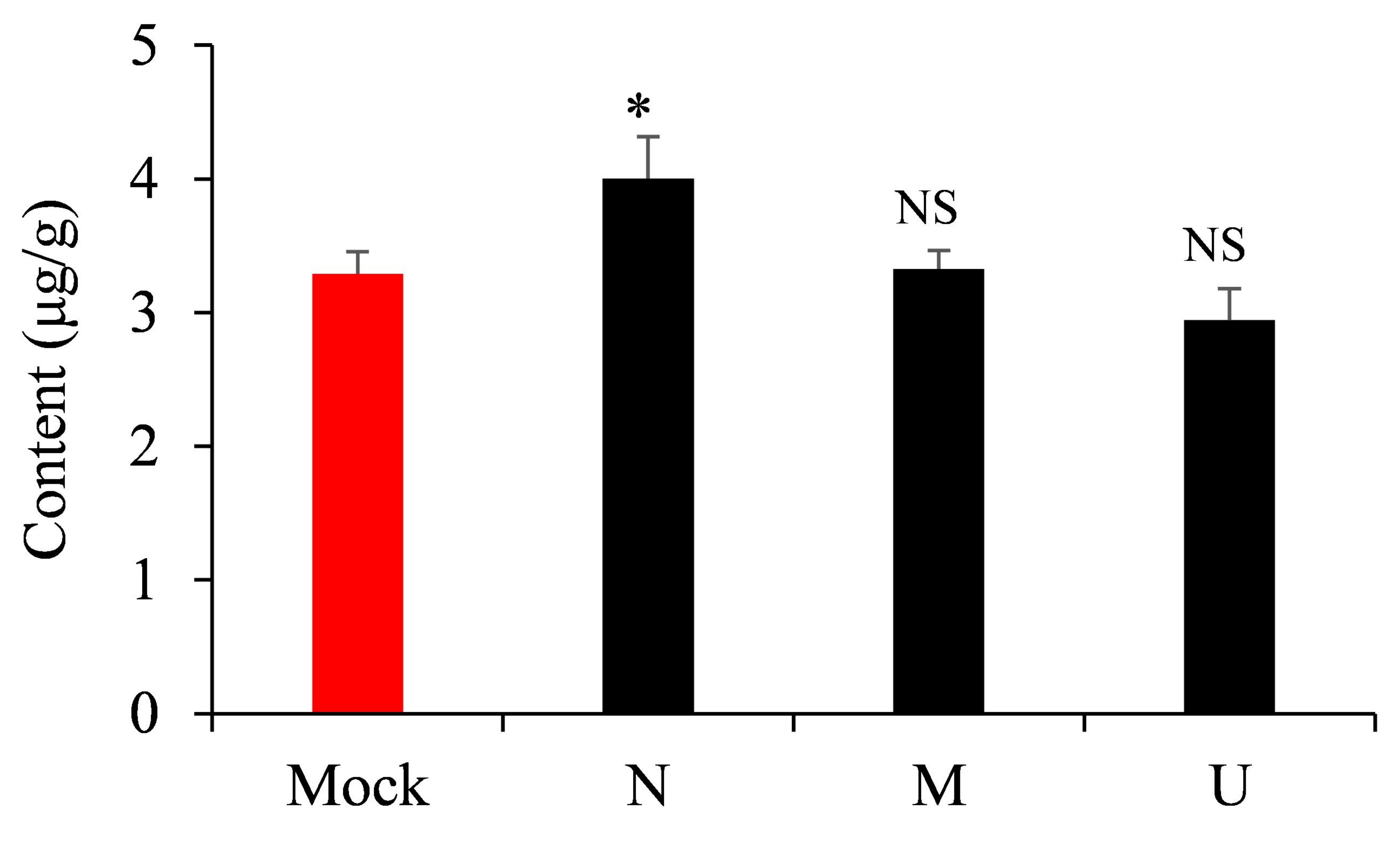

U:uninfected; M: margin; N: necrotic 
A

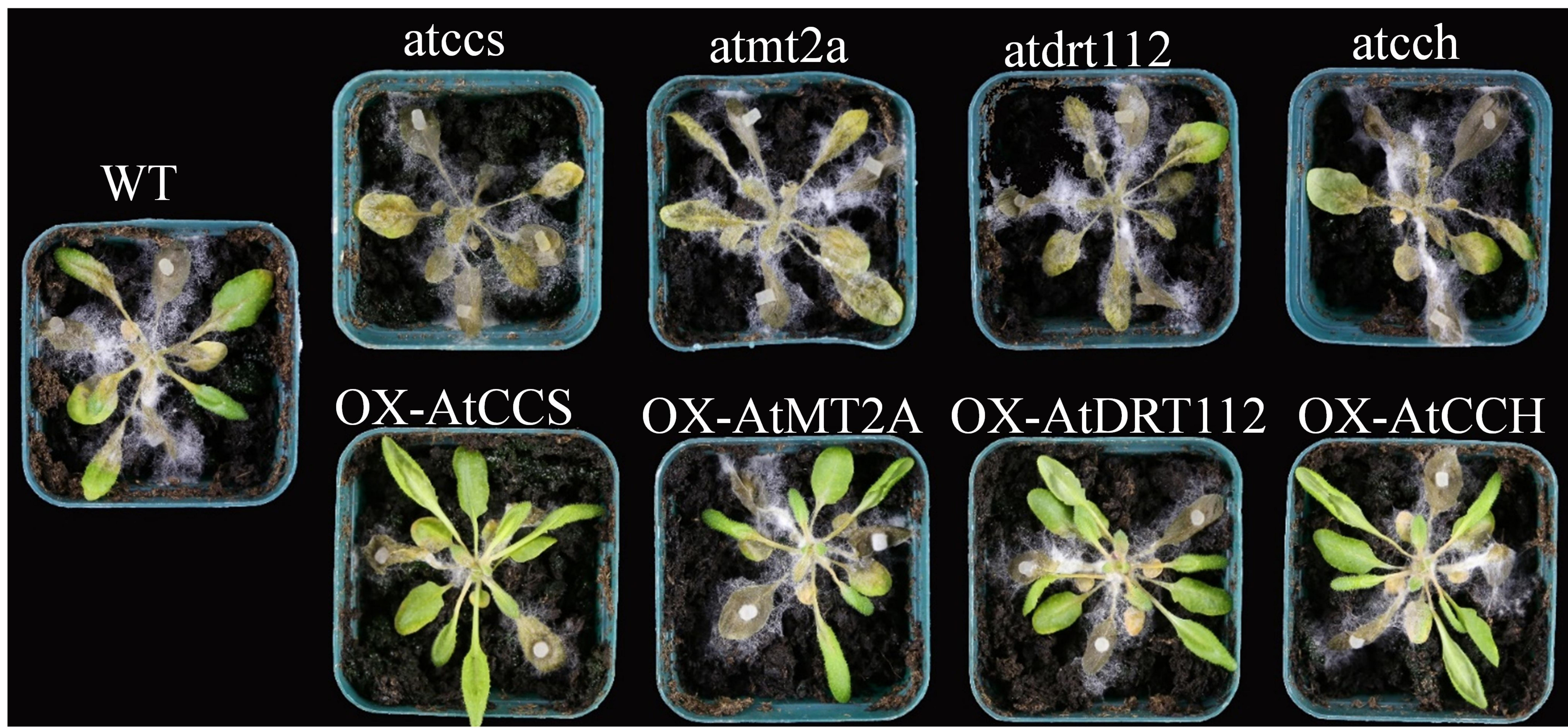

B

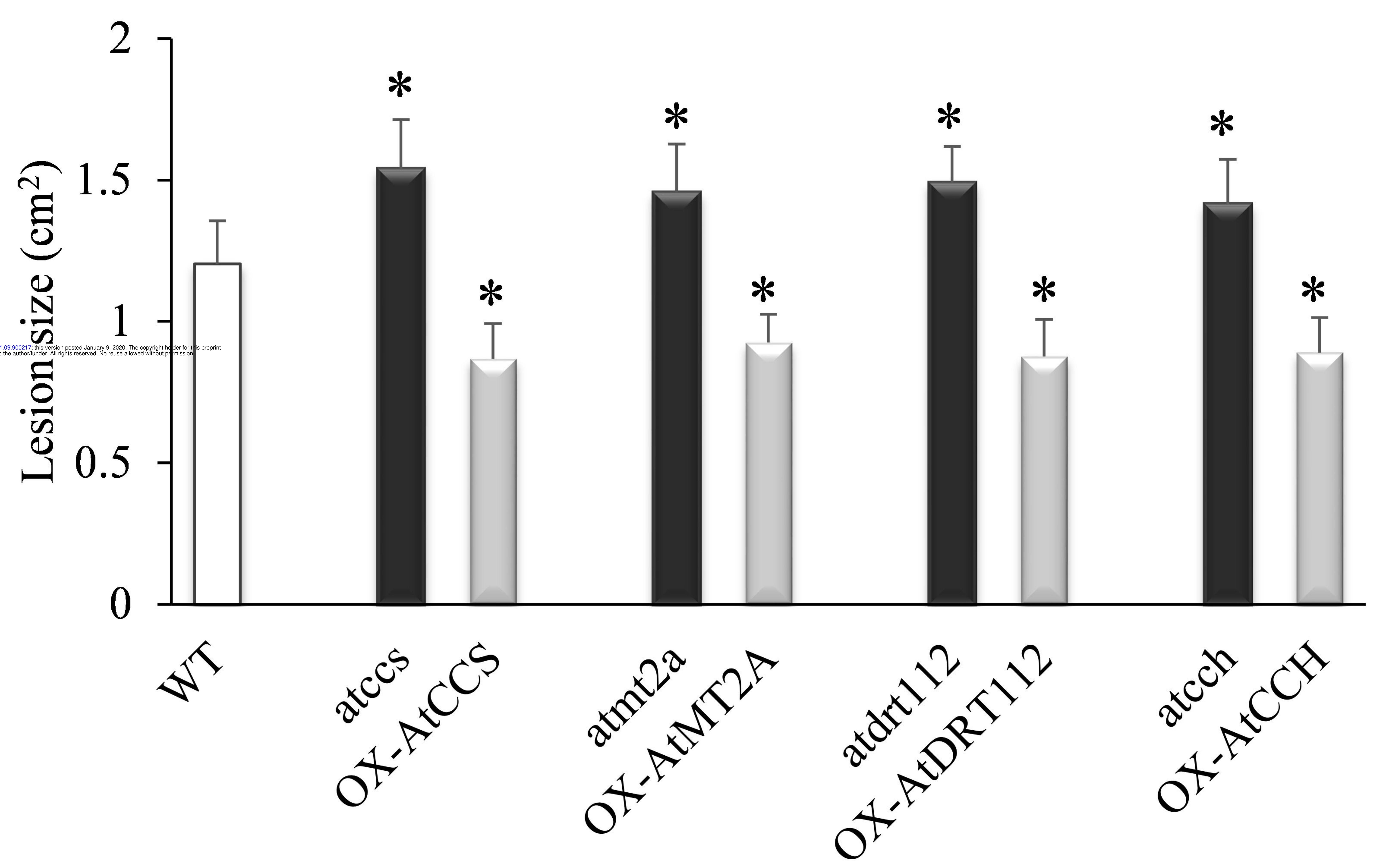


carboxylic acid catabolic process

DNA packaging

lipid catabolic process

organ senescence

response to hormone stimulus

signaling pathway

systemic acquired resistance

transport defense response response to chitin response to fungus response to oxidative stress hormone-mediated signaling pathway catabolic process

Localization

response to salicylic acid stimulus signaling




A DAB staining

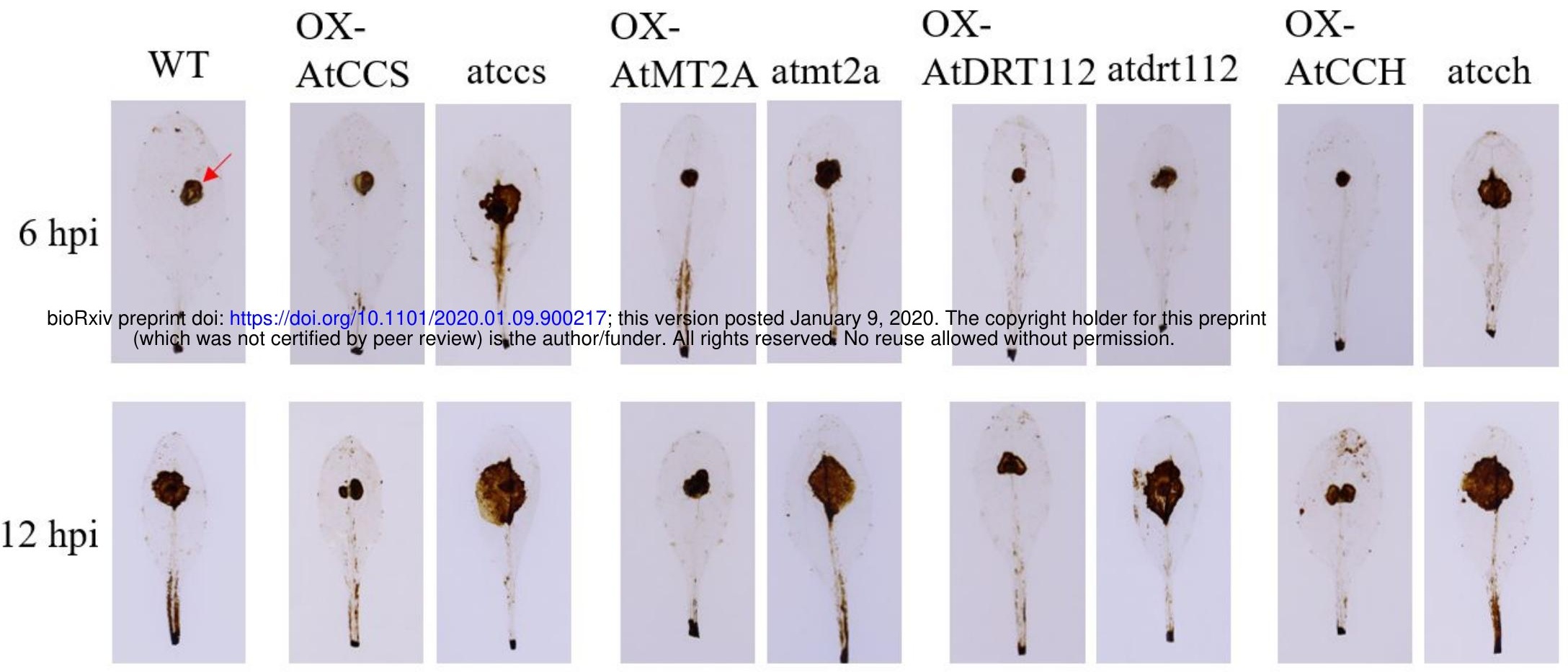

NBT staining
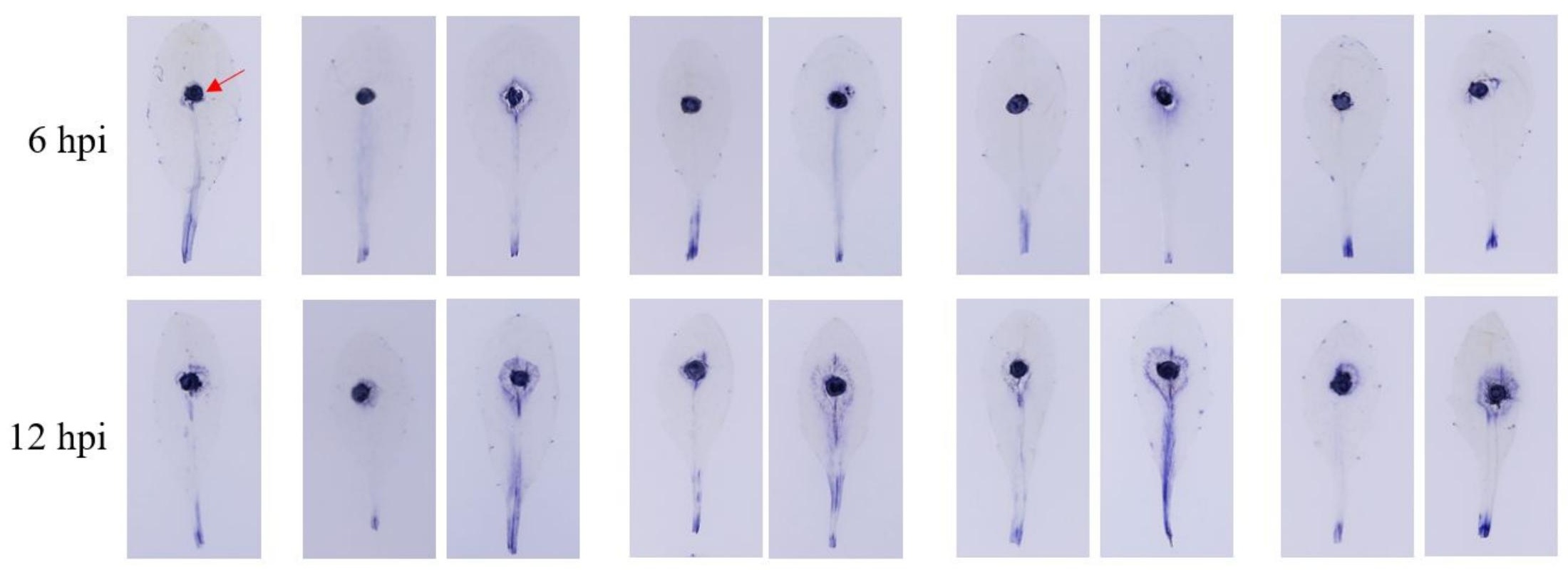

B

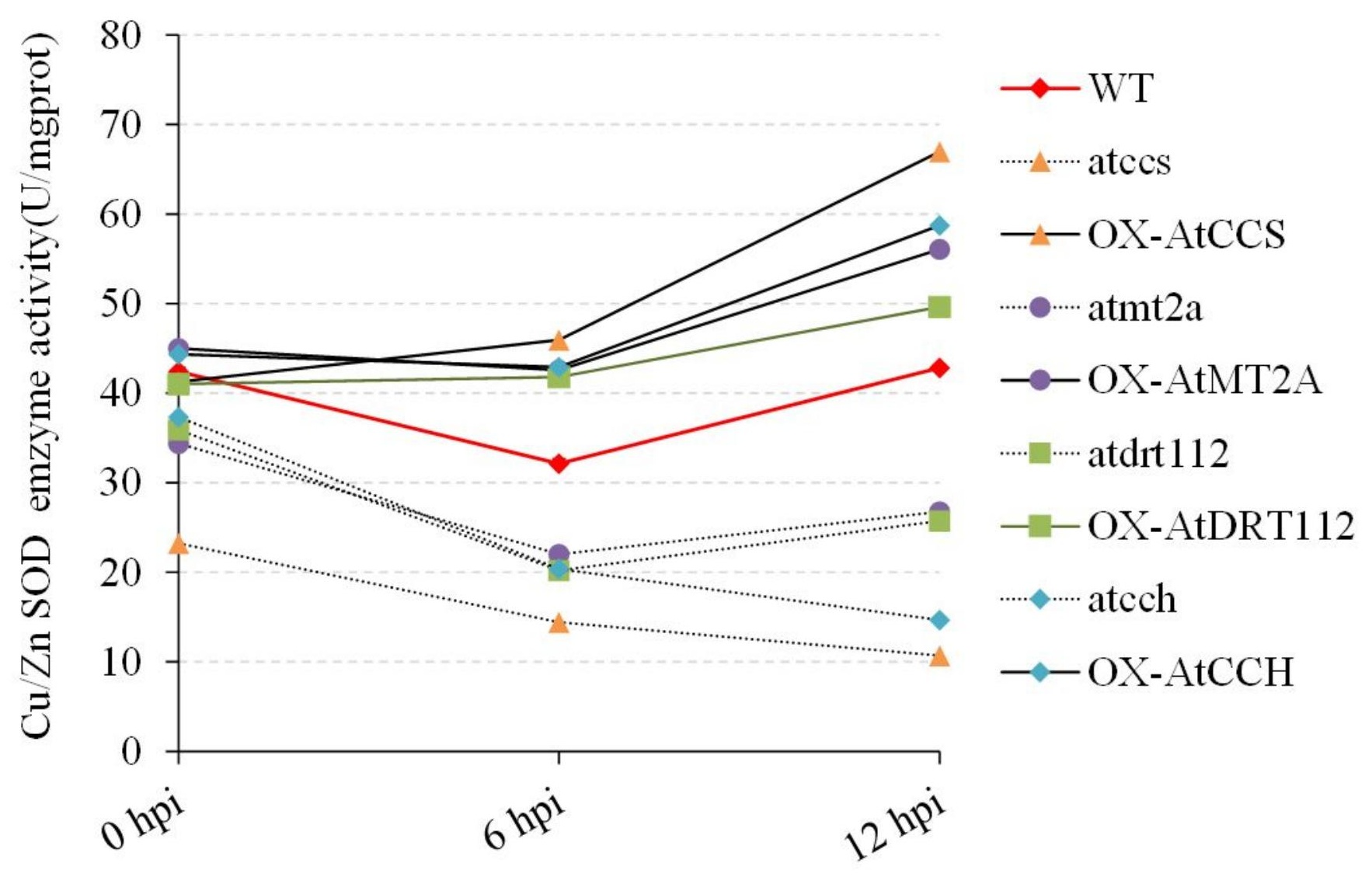


$\mathbf{A}$


B

$\square 1980 \backsim$ OXctr1 $\square$ Sictr1 $\backsim$ Oxces $\backsim$ Siccs $\backsim$ OXatx1 $\backsim$ Siatx1

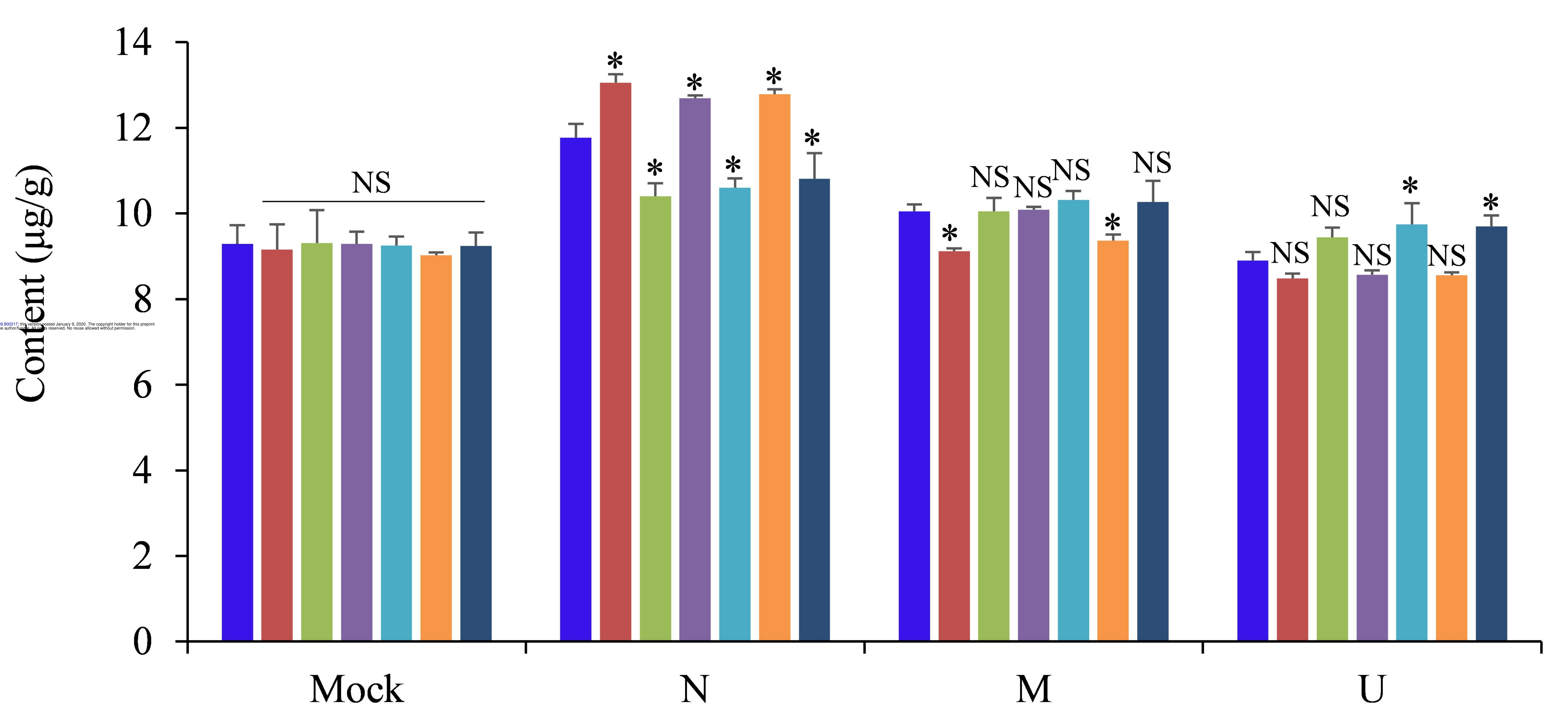




\section{Host}

\section{Resistant line}

'copper ion homeostasis' process is maintained

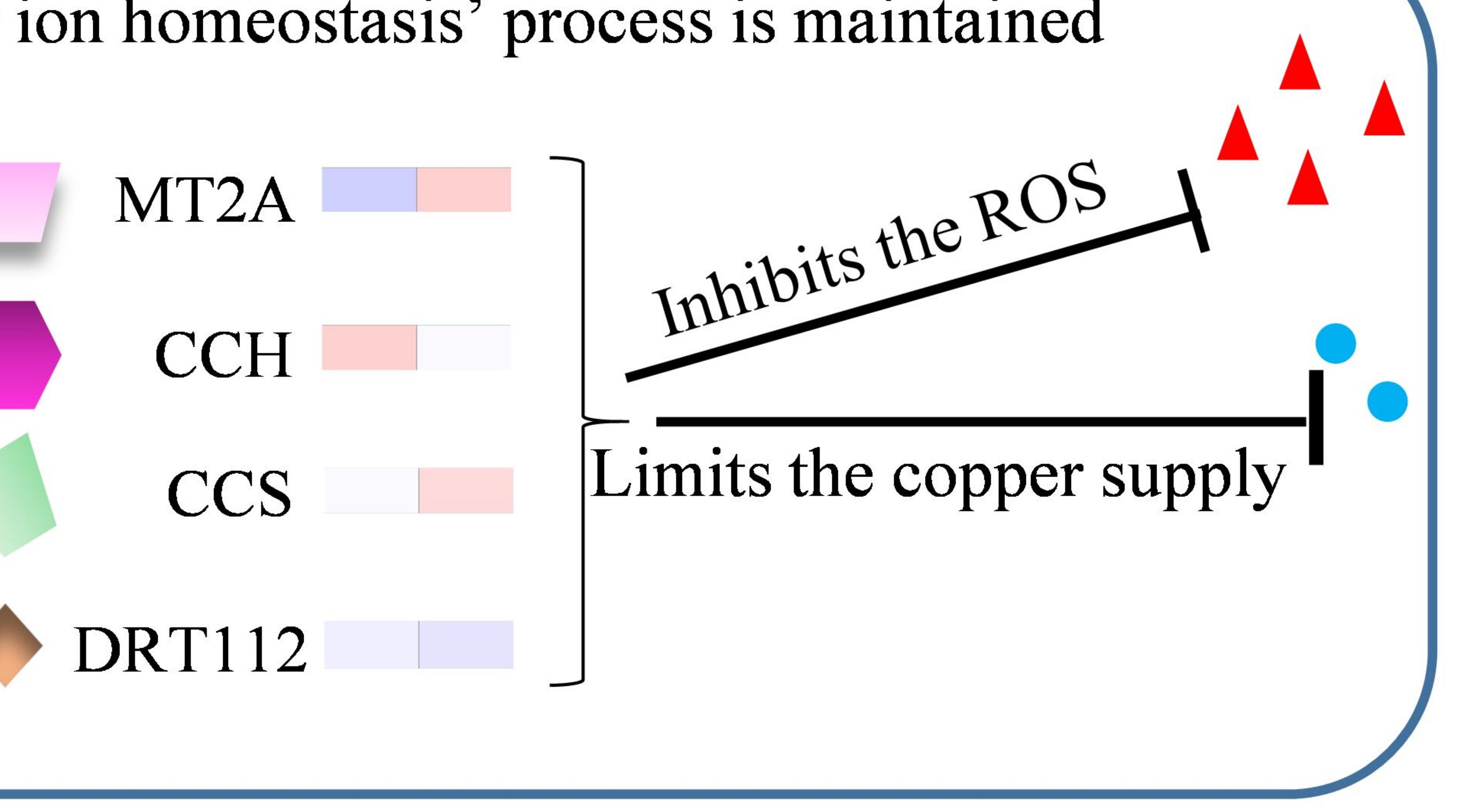

\section{Susceptible line}

'copper ion homeostasis' process is disrupted
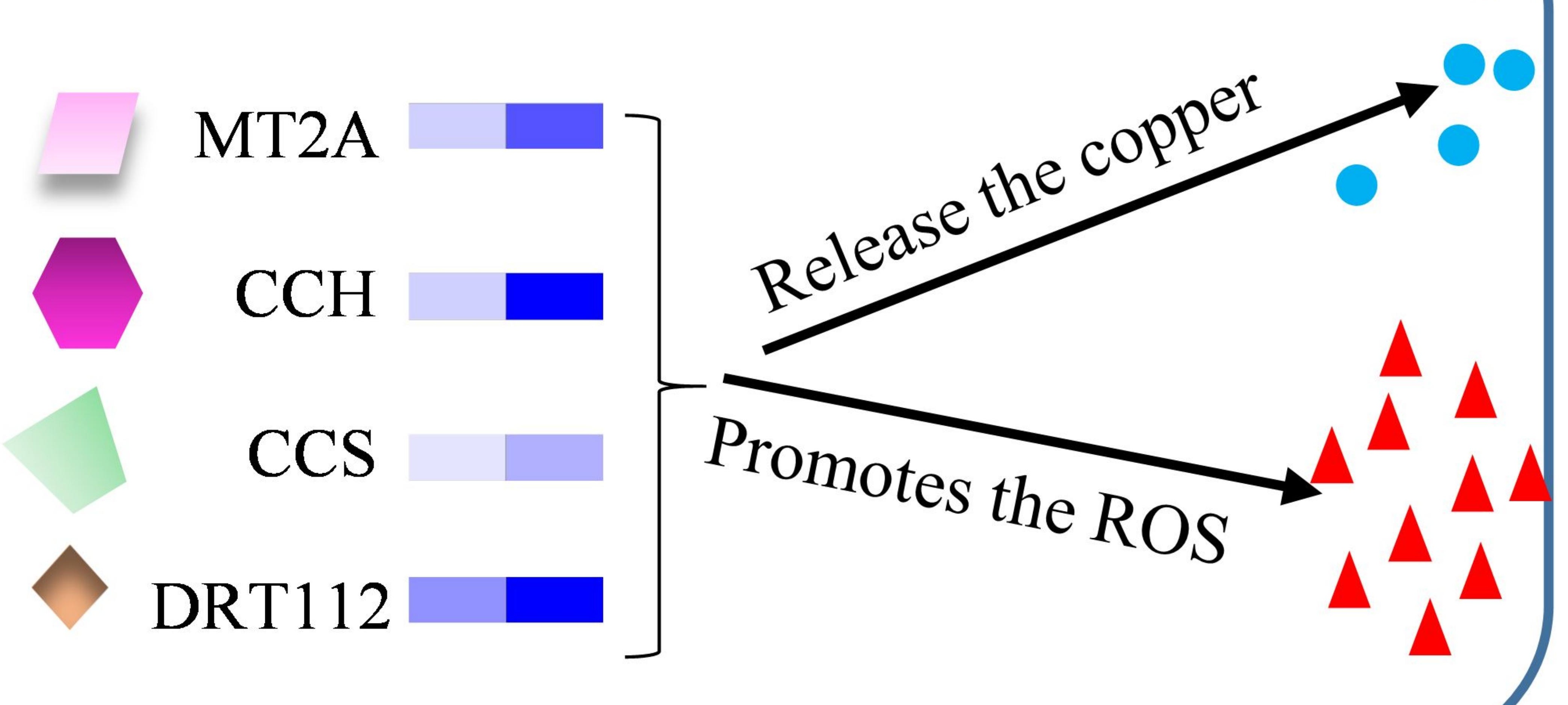

Interface

$\triangle$ ROS

- $\mathrm{Cu}^{+}$

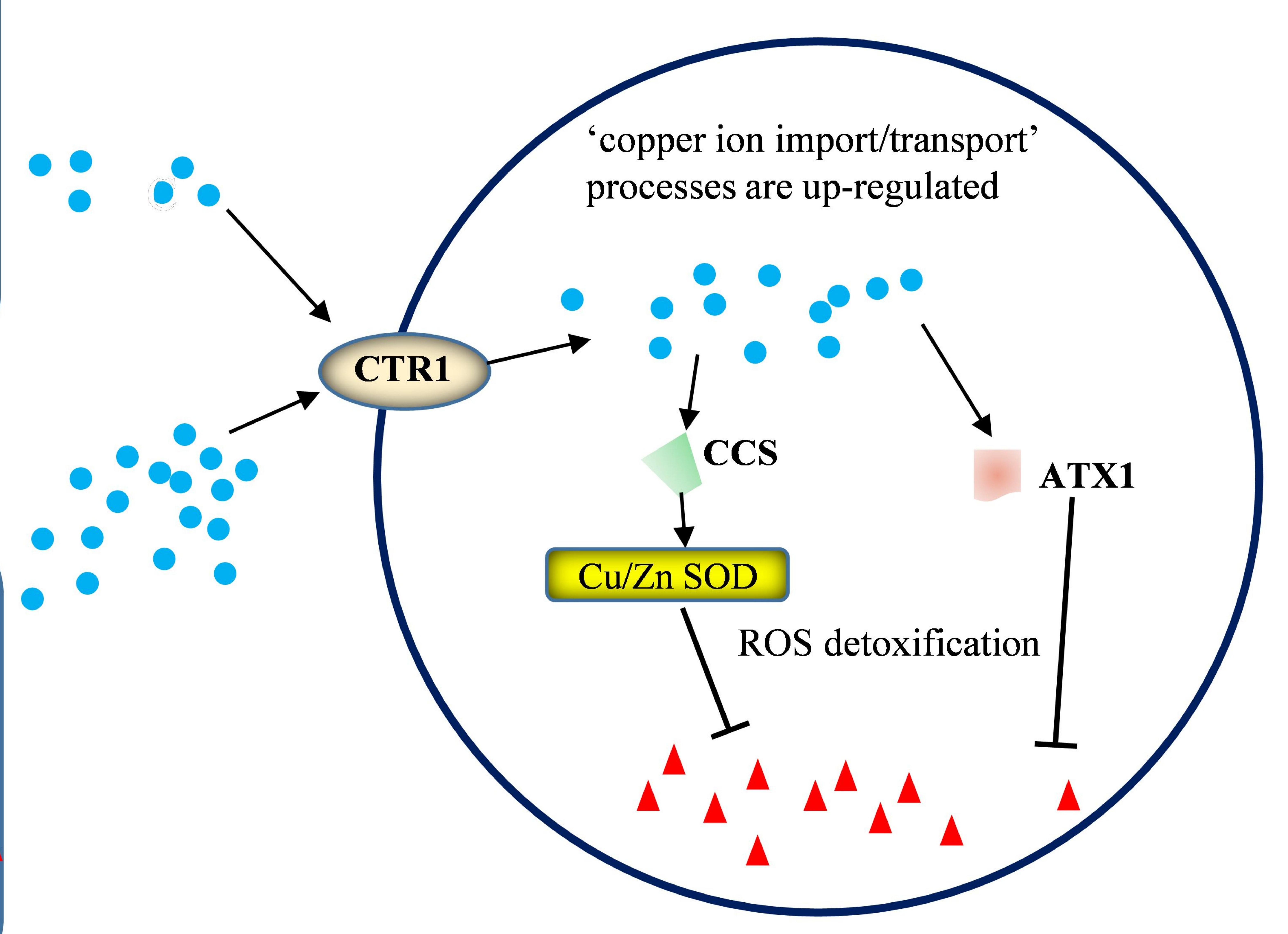

$12 / 0 \quad 24 / 12$

$$
\begin{array}{ccc}
-5 & 0 \\
& \log 2(\mathrm{FC}) & 5
\end{array}
$$

MT2A: metallothionein 2A

$\mathrm{CCH}$ : copper chaperone

CCS: copper chaperone for SOD1

DRT112: DNA-damage resistance protein 112

12/0: DEGs in B. oleracea comparing $12 \mathrm{hpi}$ to $0 \mathrm{hpi}$

24/12: DEGs in B. oleracea comparing 24 hpi to 12 hpi

CTR1: copper transport protein 1 ATX1: metal homeostasis factor 